\title{
2DOF CFD Calibrated Wake Oscillator Model to Investigate Vortex-Induced Vibrations
}

\author{
Andrey Postnikov ${ }^{1}$, Ekaterina Pavlovskaia ${ }^{1}$, and Marian Wiercigroch ${ }^{1}$ \\ 1 Centre for Applied Dynamics Research, School of Engineering, Aberdeen University, King's College, Aberdeen, AB24 3 UE, \\ Scotland, UK
}

\begin{abstract}
In this study a new two degrees-of-freedom wake oscillator model is proposed to describe vortex-induced vibrations of elastically supported cylinders capable of moving in cross-flow and in-line directions. Total hydrodynamic force acting on the cylinder is obtained here as a sum of lift and drag forces, which are defined as being proportional to the square of the magnitude of the relative flow velocity around the cylinder. The two van der Pol type oscillators are then used to model fluctuating drag and lift coefficients. As the relative velocity around the cylinder depends both on the fluid flow velocity and the velocity of the cylinder, the equations of motions of the cylinder in cross-flow and in-line directions become coupled through the fluid forces. It is shown that such approximation of the fluid forces allows to obtain the well known low dimensional models in the limit case, and the model proposed by Facchinetti et al. [1] to describe the cross-flow vibrations is used as an example. Experimental data and Computational Fluid Dynamics (CFD) results are used to calibrate the proposed model and to verify the obtained predictions of complex fluid-structure interactions for different mass ratios. A number of phenomena such as the "super-upper" branch, exclusive for a two degrees-of-freedom motion at low mass ratios, are observed. Influence of the empirical parameters of the wake oscillators equations and fluid forces coefficients on the response is also discussed.
\end{abstract}

\section{INTRODUCTION}

Slender marine structures such as risers, mooring cables, umbilicals and tethers play crucial roles in global offshore exploration, installation and production activities. As offshore oil and gas fields are moving into deeper waters, the nonlinearities in the system and the fluid-structure interaction phenomena such as vortex induced vibrations (VIVs) become more and more important. Many of VIV aspects are far from being understood and advanced modelling is required to investigate the impact of the phenomenon which significantly affects the service life of marine structures.

This work is motivated by the need of industry in effective toolkit that would allow predicting loads and fatigue damage on riser systems, especially most common Top Tensioned Risers (TTRs) and Steel Catenary Risers (SCRs), which represent a crucial part of offshore facilities. Accurate prediction of VIVs can help to produce more robust structural design and lead to substantial savings in the offshore applications.

The problem of vortex-induced vibrations is addressed by different approaches, which can be roughly categorized into one of the three major groups: experiments, computational fluid dynamics and analytical 


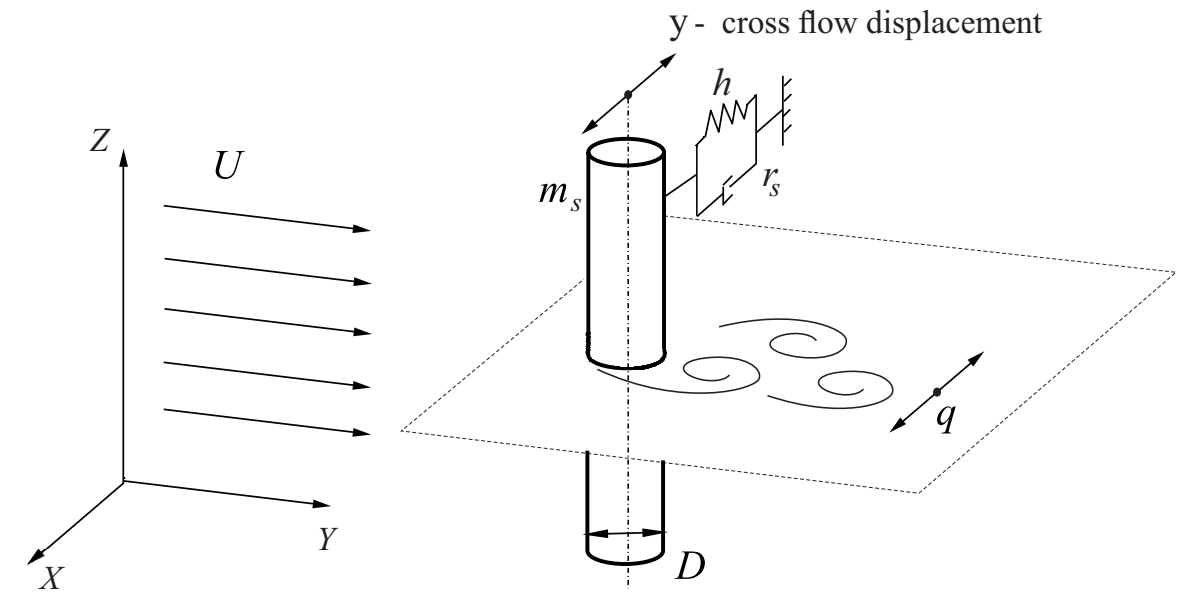

Figure 1: Wake oscillator model for the cylinder moving in transversal direction only (adopted from [1]).

models. Analytical VIV models are represented by numerous approaches to modelling both the structure and fluid, with some of them incorporating a van der Pol type equation as the governing equation for the fluid force acting on the structure. In present work, we focus on this type of analytical models, known as wake oscillator models.

The nature of the vortex shedding process behind cylindrical structure suggests that the forces acting on the structure from the fluid can be modelled by a nonlinear, self-excited oscillator called the wake oscillator. This idea was first proposed by Bishop and Hassan [2] and then investigated by Skop and Griffin [3], and Blevins and Iwan [4]. In wake oscillator models the system is usually described by two coupled ordinary differential equations. One of the equations is the equation of transversal motion of the rigid cylinder. The second equation is a semi-empirical description of fluid: the nonlinear self-excited fluid oscillator. A number of wake oscillator models were developed and applied to slender flexible structures undergoing VIV.

The Balasubramanian and Skop model [5] proposed in 1997 included a van der Pol equation driven by the local transverse motion of the cylinder as a governing equation for one component of the fluctuating lift force and a so-called stall term which is linearly proportional to the local transverse velocity of the cylinder and responsible for energy dissipation associated with motion of the cylinder in the fluid. Because of the stall term they were able to capture an asymptotic, self-limiting structural response at zero structural damping. The original form of van der Pol equation has also been reinterpreted by Krenk and Nielsen [6], Mureithi et al. [7], Plaschko [8], and Skop and Luo [9] among others.

A critical analysis on this class of low dimensional models in terms of the fundamental behaviour has been done by Facchinetti et al. [1] by considering transverse VIV of a single degree-of-freedom structure in stationary uniform flow (see Fig. 1). Three different types of coupling between structural and wake equations have been examined: via displacement, velocity, and acceleration. It was shown that the acceleration coupling provides the best match to the experimental data.

The semi-empirical approach to vortex-induced vibrations has been further studied in the work by Keber and Wiercigroch [10]. The effect of a weak structural nonlinearity on the dynamical behaviour of a vertical offshore riser undergoing VIVs was investigated, and the authors demonstrated that the structural nonlinear- 
ity has a stiffening effect on the oscillation of the riser, which becomes more pronounced when the internal flow is incorporated into the model. It was shown that additional nonlinearities in the structure affects the system significantly, and thus, for the further insight, it is important to investigate new approaches to modelling the fluid.

The new type of wake oscillator based on the Facchinetti model [1] with frequency dependent coupling was proposed by Ognik and Metrikine [11], where an attempt has been made to introduce a wake oscillator model that conforms to both the free and forced vibration experiments. Frequency dependent coupling in this case allows reproduction of the measured frequency dependence of the fluid force on the cylinder. For the time domain representation of such coupling a convolutional integral is used.

All models described so far focussed on the transversal vibrations of the structure. However the experimental investigations show that the in-line vibrations also play significant role (especially at the low mass ratios), and some attempts to develop 2DOF wake oscillator model were made, where two wake equations are used for in-line and cross-flow directions, see, for example, [12, 13, 14]. Ge et al. [12] proposed a two degrees-of-freedom wake oscillator model based on the approach by Wang et al. [15], which comes from considerations that the lift and drag forces do not coincide with $X$ and $Y$ axes (shown in Fig. 1), but act under a particular angle that indicates the direction of the cylinders instantaneous velocity. Thus, a simple force decomposition can be performed and the forces acting in $X$ and $Y$ directions estimated. Srinil and Zanganeh [13] adapted the same principle in evaluating the fluid forces but also introduced additional geometrical nonlinearities to the cylinder elastic support. These nonlinearities are included in the model by using so-called coupled Duffing oscillators, previously investigated by Raj and Rajasekar in 1997 [16]. Numerical results were tuned against existing experimental data on two degrees-of-freedom cylinders obtained by several independent researchers. In particular, results used for comparison were obtained by Jauvtis and Williamson in 2004 [17], who investigated the behaviour of low mass ratio cylinders and discovered the so-called "super-upper" branch of the response; by Stappenbelt et al. in 2007 [18], who examined behaviour of low mass ratio cylinders at very low damping ratio $\zeta=0.006$; and by Blevins and Coughran in 2009 [19], who carried on investigations on the effect of damping, varying $\zeta$ from 0.002 to 0.4 at a fixed mass ratio. The numerical results obtained by Srinil and Zanganeh demonstrated reasonable correspondence with experiments by relying on additional structural nonlinearites instead of nonlinearities provided by the fluid. Another two degree-of-feedom wake oscillator model called VIVTAS was proposed by Furnes et al. [14] for a free span pipeline undergoing VIV. In their approach they used a complex, rather than a vector, representation of the total hydrodynamic force, with one equation of motion for the structure and two nonlinear wake oscillators for real and imaginary parts of the fluid force. Preliminary model validation was performed using Marintek experimental data. In the work by Xu Bai and Wei Qin in 2014 [20], another wake oscillator model was proposed for two degrees-of-freedom VIV of elastically supported cylinder where a displacement variable related to the vortex strength was introduced. Two-dimensional potential flow approach was used in the study, with fluctuating fluid forces acting on the cylinder simplified and quantified.

Despite of recent development of these semi-empirical models focused on 2DOF motion, more work is still required to be done in the area. Many of the empirical parameters used in the existing models are evaluated using experimental studies on the stationary cylinders, where the fluid force acting on the cylinder 
is estimated using the velocity of the fluid flow. When the cylinder is elastically supported and is allowed to move in the fluid flow, the force acting on the cylinder will depend on the relative velocity of the flow around the cylinder as suggested by [21] and later by [11]. However, no existing 2DOF wake oscillator model incorporates this dependence and therefore in this paper we propose a new wake oscillator model which will fill this gap.

The rest of the paper is organised as follows. In the next section the new wake oscillator model is introduced and equations of motion are developed. The fluid forces are calculated using the relative velocity of the flow around the cylinder and oscillating lift and drag coefficients described by two van der Pol equations. Here it is shown how the damping associated with the cylinder motion in the fluid is derived from the suggested form of the fluid forces and how the proposed model could be reduced to the known model by Facchinetti [1] in the limit case where only transversal vibrations of the cylinder are considered. In the following section, the model is calibrated using first the published experimental data $[18,19,22]$ and then with our Computational Fluid Dynamics (CFD) results. The CFD model used for calculations is introduced and the results for transversal vibrations and combined transversal and in-line vibrations are used for comparison with the proposed wake oscillator model predictions. Special attention is paid to the empirical coefficients of the wake oscillators equations and their influence on the system response is investigated. Finally, some concluding remarks are given.

\section{Two degrees-of-freedom wake oscillator model}

In this work we consider an elastically supported cylinder experiencing VIV, that is free to vibrate in cross-flow and in-line directions. As mentioned in [23], for a cylinder capable of oscillating in both directions, the equations of motion on an $X Y$ plane in terms of the displacements in in-line and cross-flow directions, $x$ and $y$, are

$$
\begin{aligned}
& m^{\star} \ddot{x}+r_{s} \dot{x}+h x=F_{X}, \\
& m^{\star} \ddot{y}+r_{s} \dot{y}+h y=F_{Y},
\end{aligned}
$$

where the total hydrodynamic force components in $X$ and $Y$ directions are $F_{X}$ and $F_{Y}$. Here $m^{\star}$ is mass per unit length including an added mass $m^{\star}=m_{s}+\frac{1}{4} \pi C_{M} \rho_{f} D^{2}, r_{s}$ is structural damping, and $h$ is stiffness of the support.

This total hydrodynamic force, $\vec{F}=F_{X} \vec{i}+F_{Y} \vec{j}$, is the result of the actions of the sectional vortex-induced

$\operatorname{drag} \vec{F}_{D}$ and lift $\vec{F}_{L}$ forces which are shown in Fig. 2. As can be seen from this figure, the drag force $\vec{F}_{D}$ is acting along the velocity, $\vec{U}_{R}=\vec{U}-\vec{V}$ which is the fluid velocity relative to the cylinder [21] ( $\vec{V}$ is velocity of the cylinder and $\vec{U}=U \vec{i}$ is the velocity of the flow). The lift force $\vec{F}_{L}$ is then acting in the perpendicular directions and the magnitudes of lift and drag forces depends on the magnitude of relative velocity $\vec{U}_{R}$ as $[24]$

$$
\begin{aligned}
\vec{F}_{D} & =\frac{1}{2} \rho_{f} C_{D} D\left|\vec{U}_{R}\right|^{2} \frac{\vec{U}_{R}}{\left|\vec{U}_{R}\right|} \\
\vec{F}_{L} & =\frac{1}{2} \rho_{f} C_{L} D\left|\vec{U}_{R}\right|^{2} \mathbf{R} \cdot \frac{\vec{U}_{R}}{\left|\vec{U}_{R}\right|}
\end{aligned}
$$




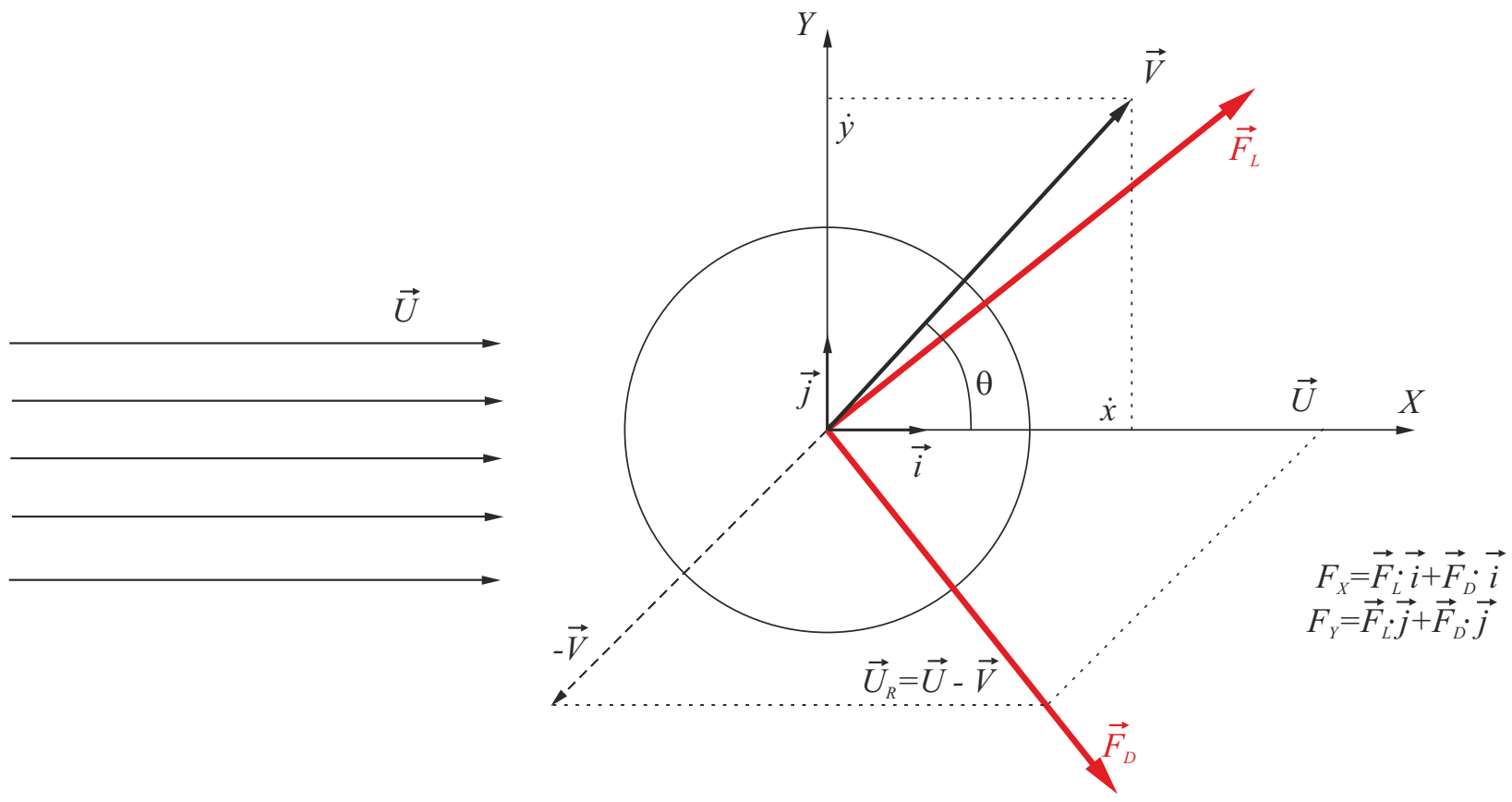

Figure 2: Forces on a vibrating cylinder with instantaneous velocity $\vec{V}$ in the flow of velocity $\vec{U}$. Drag force $\vec{F}_{D}$ acts in line with relative stream velocity $\vec{U}_{R}$.

Here to determine the direction of the lift force, the rotation tensor $\mathbf{R}=\mathbf{R}\left(\frac{\pi}{2}, \vec{k}\right)$ is used, which rotates the relative velocity vector in counterclockwise direction for $90^{\circ}$ around axis $\vec{k}$. Using Rodrigues formulation, the rotation tensor can be written as

$$
\begin{aligned}
\mathbf{R}\left(\frac{\pi}{2}, \vec{k}\right) & =\cos \left(\frac{\pi}{2}\right)(\mathbf{I}-\vec{k} \otimes \vec{k})+\sin \left(\frac{\pi}{2}\right) \vec{k} \times \mathbf{I}+\vec{k} \otimes \vec{k}= \\
& =\vec{j} \otimes \vec{i}-\vec{i} \otimes \vec{j}+\vec{k} \otimes \vec{k},
\end{aligned}
$$

where $\mathbf{I}=\vec{i} \otimes \vec{i}+\vec{j} \otimes \vec{j}+\vec{k} \otimes \vec{k}$ is the unit tensor. Substituting $\vec{U}_{R}=(U-\dot{x}) \vec{i}-\dot{y} \vec{j}$ into Eqn.(4), we obtain

$$
\vec{F}_{L}=\frac{1}{2} \rho_{f} C_{L} D\left|\vec{U}_{R}\right|^{2} \frac{\dot{y} \vec{i}+(U-\dot{x}) \vec{j}}{\left|\vec{U}_{R}\right|} .
$$

Here the other parameters are $\rho_{f}$, the fluid density, $C_{L}$, lift coefficient, and $C_{D}$, total drag, which can be represented as a sum of constant mean sectional drag $C_{D_{0}}$ and fluctuating drag, $C_{D}^{f l}$

$$
C_{D}=C_{D_{0}}+C_{D}^{f l}
$$

To determine the values of total hydrodynamic force components $F_{X}$ and $F_{Y}$, the sum of lift and total drag forces $\vec{F}=\vec{F}_{L}+\vec{F}_{D}$ should be projected on the appropriate axis (see Fig. 2) and therefore we have

$$
\begin{aligned}
& F_{X}=\left(\vec{F}_{L}+\vec{F}_{D}\right) \cdot \vec{i}, \\
& F_{Y}=\left(\vec{F}_{L}+\vec{F}_{D}\right) \cdot \vec{j},
\end{aligned}
$$


where $\vec{F}_{L}$ and $\vec{F}_{D}$ are given by Eqs.(3) and (6), and thus the projections of total hydrodynamic force $\vec{F}$ are

$$
\begin{aligned}
F_{X} & =\left(\frac{1}{2} \rho_{f} C_{L} D\left|\vec{U}_{R}\right|(\dot{y} \vec{i}+(U-\dot{x}) \vec{j})+\frac{1}{2} \rho_{f} C_{D} D\left|\vec{U}_{R}\right|((U-\dot{x}) \vec{i}-\dot{y} \vec{j})\right) \cdot \vec{i} \\
& =\frac{1}{2} \rho_{f} C_{L} D\left|\vec{U}_{R}\right| \dot{y}+\frac{1}{2} \rho_{f} C_{D} D\left|\vec{U}_{R}\right|(U-\dot{x}), \\
F_{Y} & =\left(\frac{1}{2} \rho_{f} C_{L} D\left|\vec{U}_{R}\right|(\dot{y} \vec{i}+(U-\dot{x}) \vec{j})+\frac{1}{2} \rho_{f} C_{D} D\left|\vec{U}_{R}\right|((U-\dot{x}) \vec{i} \dot{y} \vec{j})\right) \cdot \vec{j} \\
& =\frac{1}{2} \rho_{f} C_{L} D\left|\vec{U}_{R}\right|(U-\dot{x})-\frac{1}{2} \rho_{f} C_{D} D\left|\vec{U}_{R}\right| \dot{y} .
\end{aligned}
$$

In general, Eqs.(10)-(11) provide the values of the fluid forces acting on the cylinder in $X$ and $Y$ directions and these forces should be substituted in the Eqs.(1)-(2) and solved together with wake equations Eqs.(14)(15). By combining Eqs.(1)-(2) with Eqs.(10)-(11) we arrive with the system of equations describing motion of the cylinder

$$
\begin{aligned}
m^{\star} \ddot{x}+r_{s} \dot{x}+h x & =\frac{1}{2} \rho_{f} C_{L} D\left|\vec{U}_{R}\right| \dot{y}+\frac{1}{2} \rho_{f} C_{D} D\left|\vec{U}_{R}\right|(U-\dot{x}), \\
m^{\star} \ddot{y}+r_{s} \dot{y}+h y & =\frac{1}{2} \rho_{f} C_{L} D\left|\vec{U}_{R}\right|(U-\dot{x})-\frac{1}{2} \rho_{f} C_{D} D\left|\vec{U}_{R}\right| \dot{y},
\end{aligned}
$$

with $\left|\vec{U}_{R}\right|=\sqrt{(U-\dot{x})^{2}+\dot{y}^{2}}$.

Following the approach employing nonlinear oscillator equations of the van der Pol type [2] [3] [24], the fluctuating lift $C_{L}$ and $\operatorname{drag} C_{D}^{f l}$ coefficients could be modeled by two wake oscillators using $q$ and $w$ variables $\left(q=2 C_{L} / C_{L_{0}}\right.$ and $\left.w=2 C_{D}^{f l} / C_{D_{0}}^{f l}\right)$

$$
\begin{aligned}
& \ddot{w}+2 \varepsilon_{x} \Omega_{F}\left(w^{2}-1\right) \dot{w}+4 \Omega_{F}^{2} w=S_{x}, \\
& \ddot{q}+\varepsilon_{y} \Omega_{F}\left(q^{2}-1\right) \dot{q}+\Omega_{F}^{2} q=S_{y},
\end{aligned}
$$

where $\varepsilon_{x}$ and $\varepsilon_{y}$ are van der Pol parameters, $\Omega_{F}=2 \pi S t(U / D)$ is the frequency of vortex shedding and $S t$ is the Strouhal number, and $S_{x}$ and $S_{y}$ are components of total structural force $\vec{F}_{S}=S_{x} \vec{i}+S_{y} \vec{j}$ coupling wake equations with equations of cylinder motions, and $C_{L 0}$ and $C_{D_{0}}^{f l}$ are lift and fluctuating drag coefficients on a stationary cylinder. For in-line vibrations, the frequency doubling is introduced to reflect an experimentally observed phenomenon often mentioned in the literature, e.g. [25]. In next sections of this work this phenomenon will be discussed further using the CFD approach. Here the acceleration coupling is adopted as proposed by Facchinetti et al. [1], and therefore the $S_{x}$ and $S_{y}$ components are

$$
\begin{aligned}
S_{x} & =\left(A_{x} / D\right) \ddot{x}, \\
S_{y} & =\left(A_{y} / D\right) \ddot{y} .
\end{aligned}
$$

As mentioned earlier, it was demonstrated by Facchinetti et al. [1] that this type of coupling, in comparison to displacement coupling and velocity coupling, provides best results when compared to experimental data. Specifically, acceleration coupling captures lock-in domains at low mass ratios with better accuracy than the other two types of coupling.

The developed equations of motion describe the vibrations of the cylinder in the fluid flow. However, a careful calibration of the model is required and specifically empirical wake oscillators equations parameters $A_{x}, A_{y}, \varepsilon_{x}$ and $\varepsilon_{y}$ need to be found. In case of a single degree-of-freedom system, numerical results by 


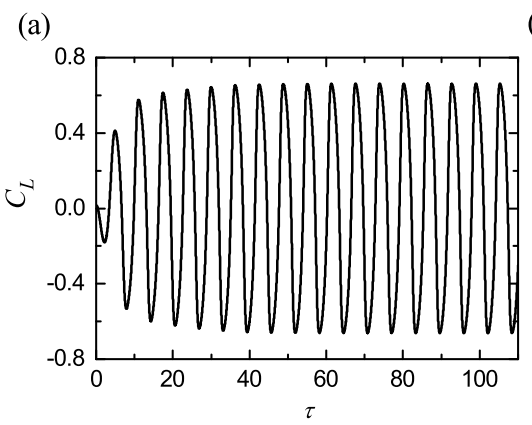

(b)

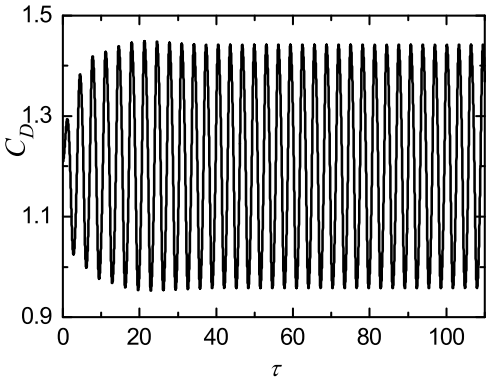

(c)

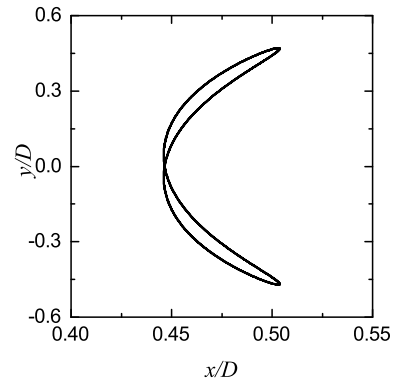

Figure 3: (a) Wake lift and (b) drag time histories, and (c) 2D cylinder trajectory for $m^{*}=1.275$ at $U_{r e d}=6.68$. $C_{D_{0}}=$ $1.2, C_{D_{0}}^{f l}=0.2, C_{L_{0}}=0.3, A_{x}=A_{y}=12, \varepsilon_{x}=\varepsilon_{y}=0.3$.

(a)

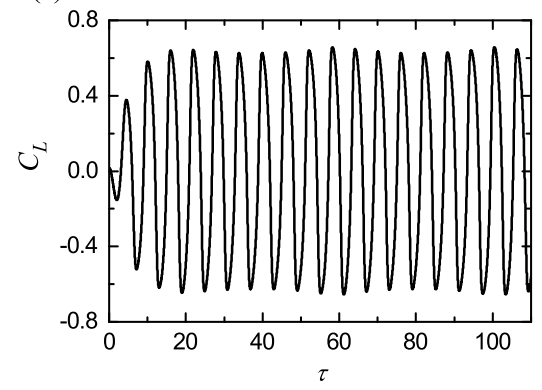

(b)

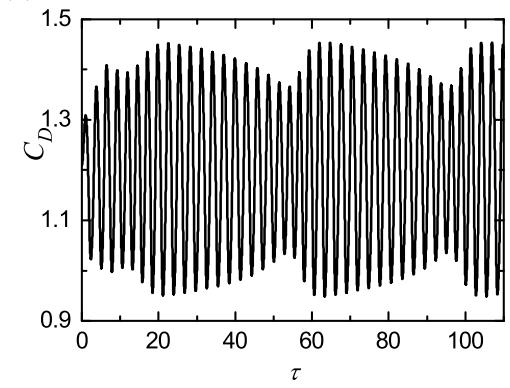

(c)

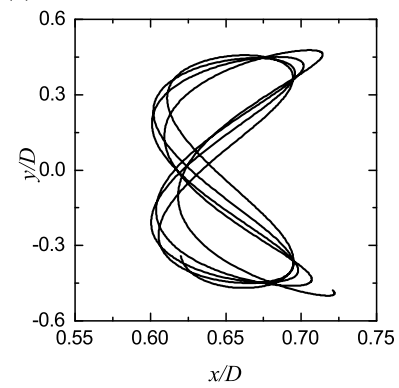

Figure 4: (a) Wake lift and (b) drag time histories, and (c) $2 \mathrm{D}$ cylinder trajectory for $m^{*}=1.275$ at $U_{\text {red }}=8.01 . C_{D_{0}}=$ $1.2, C_{D_{0}}^{f l}=0.2, C_{L_{0}}=0.3, A_{x}=A_{y}=12, \varepsilon_{x}=\varepsilon_{y}=0.3$.

Facchinetti [1] where fitted against experimental data, with $A_{y}$ and $\varepsilon_{y}$ estimated as 12 and 0.3 respectively. However, further investigation and calibration for 2DOF models are essential and will be discussed in the Section 3. It should be noted that in general these coefficients may be a function of various parameters of the system such as mass ratio, damping ratio, reduced velocity, added mass coefficient, Reynolds number, etc.

Before the model calibration is discussed, the model equations will be transformed in the non-dimensional form, and then the approximations of the fluid forces for small cylinder velocity and limit case of the transversal vibrations only will be discussed in the following subsections.

\subsection{Full two degrees-of-freedom wake oscillator model in a non-dimensional form}

The proposed full two degrees-of-freedom wake oscillator model in non-dimensional form is described by the following four second order coupled nonlinear differential equations

$$
\begin{aligned}
\tilde{x}^{\prime \prime} & +2 \zeta \tilde{x}^{\prime}+\tilde{x}=8 \pi^{2} S t^{2} \sqrt{\left(\frac{U_{\text {red }}}{2 \pi}-\tilde{x}^{\prime}\right)^{2}+\tilde{y}^{\prime 2}}\left(\frac{1}{2} M_{L} q \tilde{y}^{\prime}\right. \\
& \left.+\left(M_{D}+\frac{1}{2} M_{D}^{f l} w\right)\left(\frac{U_{r e d}}{2 \pi}-\tilde{x}^{\prime}\right)\right), \\
\tilde{y}^{\prime \prime} & +2 \zeta \tilde{y}^{\prime}+\tilde{y}=8 \pi^{2} S t^{2} \sqrt{\left(\frac{U_{\text {red }}}{2 \pi}-\tilde{x}^{\prime}\right)^{2}+\tilde{y}^{\prime 2}}\left(\frac{1}{2} M_{L} q\left(\frac{U_{\text {red }}}{2 \pi}-\tilde{x}^{\prime}\right)\right. \\
& \left.-\left(M_{D}+\frac{1}{2} M_{D}^{f l} w\right) \tilde{y}^{\prime}\right),
\end{aligned}
$$




$$
\begin{aligned}
& w^{\prime \prime}+2 \varepsilon_{x} \Omega\left(w^{2}-1\right) w^{\prime}+4 \Omega^{2} w=A_{x} \tilde{x}^{\prime \prime}, \\
& q^{\prime \prime}+\varepsilon_{y} \Omega\left(q^{2}-1\right) q^{\prime}+\Omega^{2} q=A_{y} \tilde{y}^{\prime \prime},
\end{aligned}
$$

where prime denotes differentiation with respect to non-dimensional time $\tau$ and the following variables and system parameters are introduced

$$
\begin{gathered}
\tau=\omega_{n} t, \quad \tilde{x}=x / D, \quad \tilde{y}=y / D, \\
\omega_{n}=\sqrt{h / m^{\star}}, \quad \zeta=r_{s} /\left(2 \omega_{n} m^{\star}\right), \quad \Omega=\Omega_{F} / \omega_{n}, \quad U_{r e d}=2 \pi U /\left(\omega_{n} D\right), \\
\mu=\left(m_{s}+\frac{1}{4} \pi C_{M} \rho_{f} D^{2}\right) / \rho_{f} D^{2}, \quad M_{L}=C_{L_{0}} / 16 \pi^{2} S t^{2} \mu, \quad M_{D}=C_{D_{0}} / 16 \pi^{2} S t^{2} \mu, \quad M_{D}^{f l}=C_{D_{0}}^{f l} / 16 \pi^{2} S t^{2} \mu,
\end{gathered}
$$

where $\mu$ is mass ratio in Facchinetti notation [1]. The first two equations describe the dynamics of the structure whilst the remaining two mimic the forces acting from the fluid. The relation between mass ratios in Williamson [26] and Facchinetti [1] notations, $m^{*}$ and $\mu$ correspondingly, is described by

$$
m^{*}=4 \mu / \pi-C_{M} .
$$

Figs. 3 and 4 present two examples of system response calculated using this model for $U_{\text {red }}=6.68$ and $U_{\text {red }}=8.01$, respectively. Parts (a) and (b) show the lift and drag coefficient time histories and parts (c) demonstrate $2 \mathrm{D}$ cylinder trajectories computed for $m^{*}=1.275$. In this case the wake oscillators coefficients are chosen to be the same as in [1] for $1 \mathrm{D}$ case, i.e. $A_{x}=A_{y}=12$ and $\varepsilon_{x}=\varepsilon_{y}=0.3$.

\subsection{Approximate two degrees-of-freedom wake oscillator model}

In order to compare the proposed model with the models available in the literature we will use some commonly accepted assumptions. Assuming that the horizontal and vertical velocities of the cylinder, $\dot{x}$ and $\dot{y}$, are smaller than the magnitude of the flow velocity $U=|\vec{U}|[15]$, and introducing notation $U_{R}=\left|\vec{U}_{R}\right|$, we can approximate the value of the relative velocity using the following expansion

$$
U_{R}=\sqrt{(U-\dot{x})^{2}+\dot{y}^{2}}=U \sqrt{\left(1-\frac{\dot{x}}{U}\right)^{2}+\left(\frac{\dot{y}}{U}\right)^{2}} \approx U\left(1-\frac{\dot{x}}{U}+\frac{1}{2} \frac{\dot{y}^{2}}{U^{2}}\right) .
$$

Substituting $U_{R}$ into the equations for $F_{X}$ and $F_{Y}$ we obtain

$$
\begin{aligned}
& F_{X}=\frac{1}{2} \rho_{f} C_{L} D U\left(\dot{y}-\frac{\dot{x} \dot{y}}{U}\right)+\frac{1}{2} \rho_{f} C_{D} D U\left(U-2 \dot{x}+\frac{\dot{y}^{2}}{2 U}+\frac{\dot{x}^{2}}{U}\right), \\
& F_{Y}=\frac{1}{2} \rho_{f} C_{L} D U\left(U-2 \dot{x}+\frac{\dot{y}^{2}}{2 U}+\frac{\dot{x}^{2}}{U}\right)-\frac{1}{2} \rho_{f} C_{D} D U\left(\dot{y}-\frac{\dot{x} \dot{y}}{U}\right) .
\end{aligned}
$$

Now we can substitute $\dot{x}=\epsilon \dot{\bar{x}}, \dot{y}=\epsilon \dot{\bar{y}}, C_{L}=\epsilon \bar{C}_{L}, C_{D}^{f l}=\epsilon \bar{C}_{D}^{f l}$, where $\dot{\bar{x}}, \dot{\bar{y}}, \bar{C}_{L}, \bar{C}_{D}^{f l}$ are no longer small, and rewrite expressions for forcing terms as follows

$$
\begin{aligned}
F_{X} & =\frac{1}{2} \rho_{f} D\left(C_{D_{0}} U^{2}+\epsilon\left(\bar{C}_{D}^{f l} U^{2}-2 C_{D_{0}} U \dot{\bar{x}}\right)+\epsilon^{2}\left(\bar{C}_{L} U \dot{\bar{y}}+\frac{1}{2} C_{D_{0}} \dot{\bar{y}}^{2}+C_{D_{0}} \dot{\bar{x}}^{2}-2 \bar{C}_{D}^{f l} U \dot{\bar{x}}\right)\right. \\
& \left.+\epsilon^{3}\left(\frac{1}{2} \bar{C}_{D}^{f l} \dot{\bar{y}}^{2}+\bar{C}_{D}^{f l} \dot{\bar{x}}^{2}-\bar{C}_{L} \dot{\bar{x}} \dot{\bar{y}}\right)\right), \\
F_{Y} & =\frac{1}{2} \rho_{f} D\left(\epsilon\left(\bar{C}_{L} U^{2}-C_{D_{0}} U \dot{\bar{y}}\right)+\epsilon^{2}\left(C_{D_{0}} \dot{\bar{x}} \dot{\bar{y}}-\bar{C}_{D}^{f l} U \dot{\bar{y}}-2 \bar{C}_{L} U \dot{\bar{x}}\right)\right. \\
& \left.+\epsilon^{3}\left(\frac{1}{2} \bar{C}_{L} \dot{\bar{y}}^{2}+\bar{C}_{L} \dot{\bar{x}}^{2}+\bar{C}_{D}^{f l} \dot{\bar{x}} \dot{\bar{y}}\right)\right) .
\end{aligned}
$$


Analysing the obtained equations, we can see that that the largest force acting on the cylinder is the constant horizontal force $\frac{1}{2} \rho_{f} D C_{D_{0}} U^{2}$ associated with the constant mean sectional component of the drag. It is also clear from these equations that there are forces of the same magnitude of order of $\epsilon$ acting in both horizontal and vertical direction.

It can be noted that equations of motion contain a number of nonlinear terms, and terms $\rho_{f} D U C_{D_{0}} \dot{x}$ and $\frac{1}{2} \rho_{f} D U C_{D_{0}} \dot{y}$ representing damping from the fluid in in-line and cross-flow equations of motion correspondingly. For in-line equation of motion this damping coefficient is of a double magnitude in comparison to the one in cross-flow equation, which is an interesting feature of the proposed model, not previously captured by existing two degrees-of-freedom models $[15,13]$.

As can be seen from Eqs. (10)-(11) and also Eqs. (26)-(27), components of the total hydrodynamic force on the cylinder, containing contributions from both the lift and drag, form the right hand side of the cylinder equations representing the motion in $X Y$ plane. By omitting all terms with the order of $\epsilon^{3}$ in Eqs. (26)-(27), motion equations for this model can be written as

$$
\begin{aligned}
m^{\star} \ddot{x} & +r_{s} \dot{x}+h x=\frac{1}{2} \rho_{f} D U^{2} C_{D_{0}}+\frac{1}{4} \rho_{f} D U^{2} C_{D_{0}}^{f l} w-\rho_{f} D U C_{D_{0}} \dot{x} \\
& +\frac{1}{4} \rho_{f} D U C_{L_{0}} q \dot{y}+\frac{1}{4} \rho_{f} D C_{D_{0}} \dot{y}^{2}+\frac{1}{2} \rho_{f} D C_{D_{0}} \dot{x}^{2}-\frac{1}{2} \rho_{f} D U C_{D_{0}}^{f l} w \dot{x}, \\
m^{\star} \ddot{y} & +r_{s} \dot{y}+h y=\frac{1}{4} \rho_{f} D U^{2} C_{L_{0}} q-\frac{1}{2} \rho_{f} D U C_{D_{0}} \dot{y}+\frac{1}{2} \rho_{f} D C_{D_{0}} \dot{x} \dot{y} \\
& -\frac{1}{4} \rho_{f} D U C_{D_{0}}^{f l} w \dot{y}-\frac{1}{2} \rho_{f} D U C_{L_{0}} q \dot{x},
\end{aligned}
$$

with wake equations as follows

$$
\begin{aligned}
& \ddot{w}+2 \Omega_{F} \varepsilon_{x}\left(w^{2}-1\right) \dot{w}+4 \Omega_{F}^{2} w=\left(A_{x} / D\right) \ddot{x}, \\
& \ddot{q}+\varepsilon_{y} \Omega_{F}\left(q^{2}-1\right) \dot{q}+\Omega_{F}^{2} q=\left(A_{y} / D\right) \ddot{y} .
\end{aligned}
$$

If the motion of the cylinder in vertical (in-line) direction is omitted $(\dot{x}=0)$ and the fluctuating drag is ignored, keeping only terms of order of $\epsilon$ we can obtain one degree-of-freedom wake oscillator equation which is widely used (see for example [1]) with $F_{Y}$ including the wake force term and the stall term (damping associated with the fluid motion)

$$
F_{Y}=\frac{1}{2} \rho_{f} C_{L} D U^{2}-\frac{1}{2} \rho_{f} C_{D_{0}} D U \dot{y}
$$

where $C_{L}=C_{L_{0}} q / 2$ and $C_{L 0}$ is usually taken as 0.3 . Then one degree-of-freedom wake oscillator model will look as follows

$$
\begin{aligned}
& \left(m_{s}+\frac{1}{4} \pi C_{M} \rho_{f} D^{2}\right) \ddot{y}+r_{s} \dot{y}+h y=\frac{1}{4} \rho_{f} D U^{2} C_{L_{0}} q-\frac{1}{2} \rho_{f} D U C_{D_{0}} \dot{y}, \\
& \ddot{q}+\varepsilon_{y} \Omega_{F}\left(q^{2}-1\right) \dot{q}+\Omega_{F}^{2} q=\left(A_{y} / D\right) \ddot{y} .
\end{aligned}
$$

The analysis presented in this sub-section demonstrates that by using widely accepted assumption of the small velocity of the cylinder, the proposed model could be reduced to the existing models in the limit case of transversal vibrations only, naturally obtaining both well-known fluid force damping term (e.g. stall term) and the appropriate lift force term.

In the next section, the calibration of the model will be considered and the responses obtained using the new model will be compared with published experimental data and also with results of the CFD analysis. 

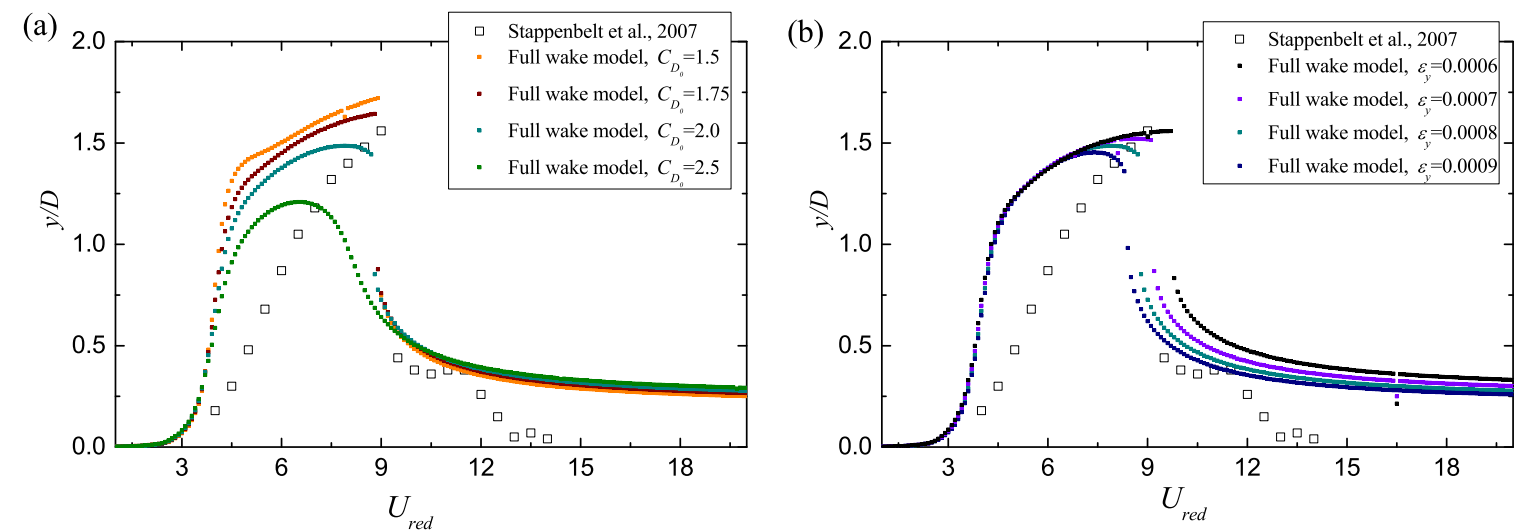

Figure 5: Resonance curves (dependence of response amplitude on the reduced velocity). New 2DOF wake oscillator model compared to experimental data by Stappenbelt et al. [18]. $m^{*}=2.36, \zeta=0.006$. Wake parameters are $A_{y}=5, A_{x}=12$, $\varepsilon_{x}=0.3$. Reference fluid force parameters are $C_{L_{0}}=0.3, C_{D_{0}}^{f l}=0.2$. (a) Influence of mean sectional drag $C_{D_{0}}, \varepsilon_{y}=0.008$; (b) Influence of wake parameter $\varepsilon_{y}$ at $C_{D_{0}}=2.0$.
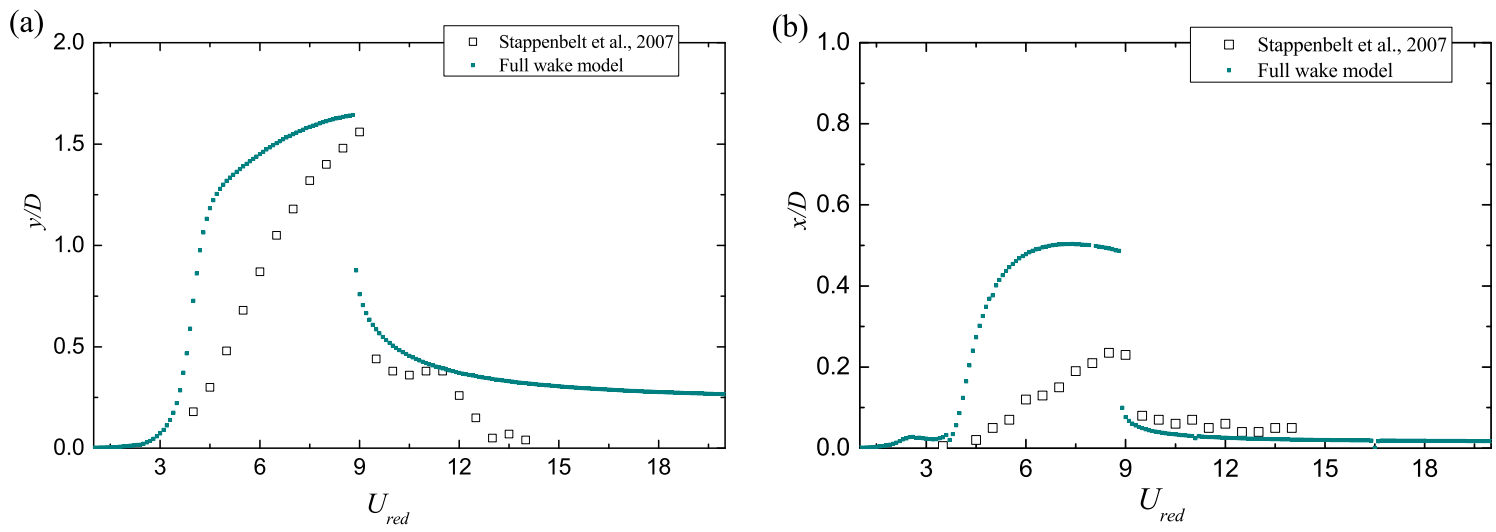

Figure 6: Resonance curves (dependence of response amplitude on the reduced velocity). New 2DOF wake oscillator model compared to experimental data by Stappenbelt et al. [18]. $m^{*}=2.36, \zeta=0.006$. Wake parameters are $A_{y}=5, A_{x}=12$, $\varepsilon_{y}=0.0008, \varepsilon_{x}=0.3$. Reference fluid force parameters are $C_{D_{0}}=1.75, C_{L_{0}}=0.3, C_{D_{0}}^{f l}=0.2$. (a) Cross-flow response; (b) In-line response.

\section{MODEL CALIBRATION USING CFD AND EXPERIMENTAL RESULTS}

\subsection{Calibration based on experimental data}

First, the published experimental results are utilised to calibrate the proposed wake oscillator model. Three sets of experimental data were considered, and comparisons are made for different mass-damping ratio parameters. As observed by Jauvtis and Williamson in experiments carried out in 2004 [17], an additional branch of response appears at $m^{*}<6$. As low mass ratios are of a particular interest in two degrees-of-freedom case, two sets of data are chosen to specifically capture the "super-upper" branch, at $m^{*}=2.36$ by Stappenbelt et al. [18] and $m^{*}=2.6$ by Jauvtis and Williamson [17, 22].

Figure 5 presents the amplitude of the transversal vibrations as function of the flow velocity $\left(U_{\text {red }}=\right.$ $\left.2 \pi U /\left(D \omega_{n}\right)\right)$. The amplitude of transversal vibration is calculated as a maximum value of cylinder displacement at a given value of $U_{\text {red }}$. Here the system responses are calculated using the proposed model for different values of the drag coefficient $C_{D_{0}}$ in part (a) and different values of wake oscillator coefficients $\varepsilon_{y}$ in part (b). In general, choosing the wake oscillator parameters is a challenging task and in the future 
(a)

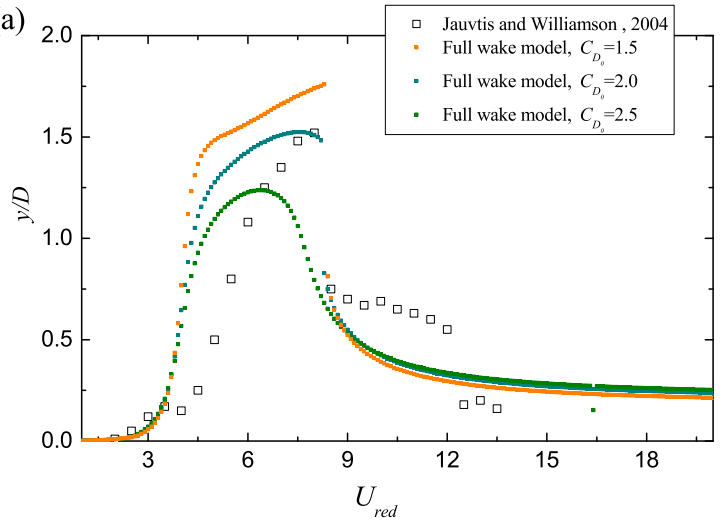

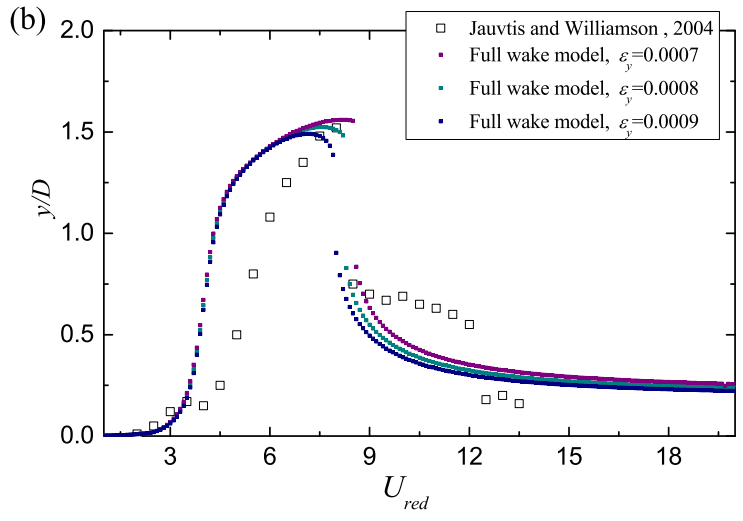

Figure 7: Resonance curves (dependence of response amplitude on the reduced velocity). New 2DOF wake oscillator model compared to experimental data by Jauvtis and Williamson [22]. $m^{*}=2.6, \zeta=0.0036$. Wake parameters are $A_{y}=5, A_{x}=12$, $\varepsilon_{x}=0.3$. Reference fluid force parameters are $C_{L_{0}}=0.3, C_{D_{0}}^{f l}=0.2$. (a) Influence of mean sectional drag $C_{D_{0}}, \varepsilon_{y}=0.008$; (b) Influence of wake parameter $\varepsilon_{y}$ at $C_{D_{0}}=2.0$

this could be done using comprehensive optimisation procedure. However, some preliminary tuning of the parameters can be done without it, and the results are shown in Fig. 5 where amplitudes evaluated using the tuned wake oscillator model are compared to experimental data by Stappenbelt et al. [18] $\left(m^{*}=2.36\right.$, $\zeta=0.006$ ). In order to achieve a reasonable match, both the coupling coefficient $A_{y}$ and the wake oscillator coefficient $\varepsilon_{y}$ were reduced to $A_{y}=5$ and $\varepsilon_{y}=0.008 . C_{D_{0}}$ is normally taken as a constant value, estimated the same way as other reference parameters, however this assumption neglects the fact that value of $C_{D_{0}}$ depends on the transverse amplitude of vibration. As can be seen in Fig. 5a, mean sectional drag affects the branch of response significantly. Recommended value for this parameter is $C_{D_{0}}=2.0$ if it is not chosen to be modelled as a function of transversal amplitude for the sake of simplicity. It can be noted here, that alternation of this parameter can vastly change the shape of response branch, specifically to nullify the jump to the lower branch of response as illustrated in Fig. 5a for the value $C_{D_{0}}=2.5$.

Fig. 5b demonstrates the best fit at tuned van der Pol parameter $\varepsilon_{y}$. A fairly good fit to experimental data is observed at $0.007<\varepsilon_{y}<0.009$. Overall, tuning $\varepsilon_{y}$ has given best results in fitting the response curve to experimental data, although generally a combined $A_{y} / \varepsilon_{y}$ parameter must be considered.

The best fit at $C_{D_{0}}=1.75$ (Fig. 6a) was chosen to illustrate the in-line amplitude predictions, which are presented in Fig. 6b. It can be seen that in-line amplitudes are higher than those observed experimentally. Our analysis indicates that adjusting parameters of the wake oscillator equation in in-line direction $A_{x}$ and $\varepsilon_{x}$ does not have the same effect on the in-line vibration amplitudes as parameters $A_{y}$ and $\varepsilon_{y}$ on the transversal amplitudes. It is clear that more in-depth investigation is required to achieve a better match between the model predictions and the experimental data. However, even for this non-optimised choice of parameters, the overestimation of the in-line amplitude could be acceptable if the model is to be used for the design calculations as satisfying the design criteria in this case will improve overall safety factor.

Fig. 7 presents comparisons with experiments by Jauvtis and Williamson [22] at a slightly higher mass ratio $m^{*}=2.6$ and lower damping ratio $\zeta=0.0015$. It can be noted here that the same recommendations apply for both amplified sectional $\operatorname{drag} C_{D_{0}}$ and wake parameter $\varepsilon_{y}$ since the presented results demonstrate similar trends for both sets of experimental data. The main issue, common for both cases, is that the 
(a)

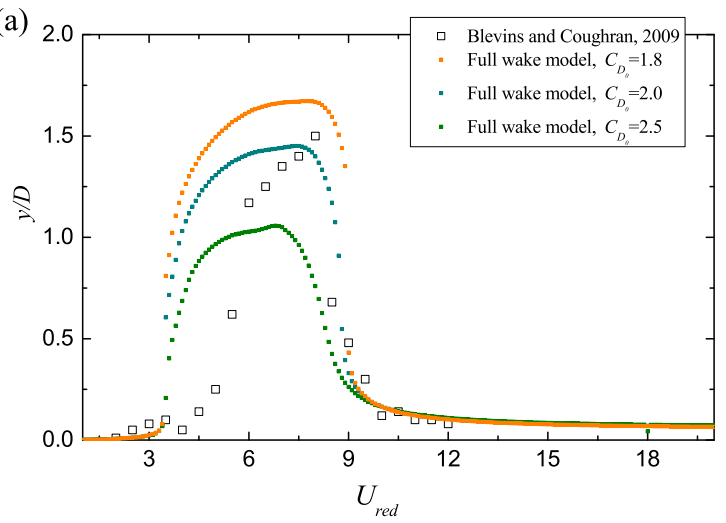

(b)

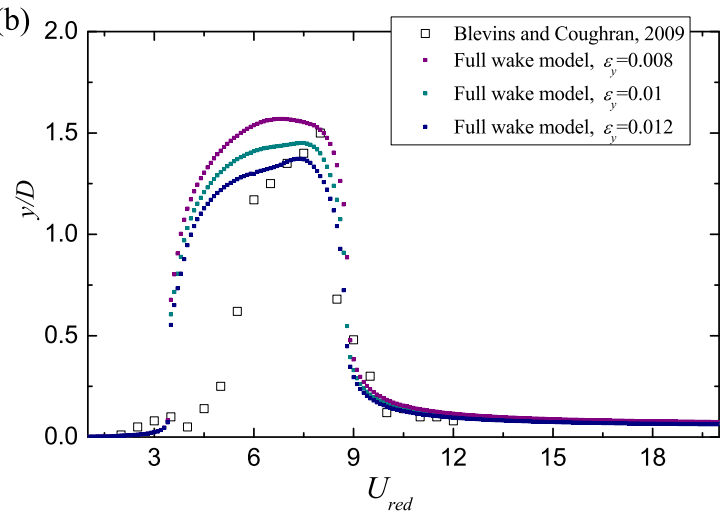

Figure 8: Resonance curves (dependence of response amplitude on the reduced velocity). New 2DOF wake oscillator model compared to experimental data by Blevins and Coughran [19]. $m^{*}=5.4, \zeta=0.002$. Wake parameters are $A_{y}=24, A_{x}=12$, $\varepsilon_{x}=0.3$. Reference fluid force parameters are $C_{L_{0}}=0.3, C_{D_{0}}^{f l}=0.2$. (a) Influence of mean sectional drag $C_{D_{0}}, \varepsilon_{y}=0.01 ;(\mathrm{b})$ Influence of wake parameter $\varepsilon_{y}$ at $C_{D_{0}}=2.0$

upper branch of the calculated response always happens to be shifted to the left giving overestimation of the amplitude values at lower reduced velocities in the lock-in region.

Finally, a comparison with experimental data by Blevins and Goughran [19] for mass ratio $m^{*}=5.4$ is presented in Fig. 8. A notable difference from other presented experimental data is the absence of a lower branch after an amplitude drop around $U_{\text {red }} \approx 8$. The response predicted by the wake oscillator model in this case shows a better correspondence with experiments, although higher values of amplitude starting at $U_{\text {red }} \approx 3.5$ were obtained indicating, similar to previously presented results, that according to the model the entrance to the lock-in region occurs at lower values of reduced velocity than in experiments.

It has to be noted that experimental data presented here were obtained using test rigs that inevitably differ from each other in their technical characteristics and may include structural nonlinearities which are not incorporated in the proposed wake oscillator model. Further experimental studies would be useful for more refined model calibration to achieve a better match and to formulate clear recommendations on the selection of the empirical wake oscillator coefficients. However, from the conducted analysis, it could be concluded that much lower values of the parameter $\varepsilon_{y}$ should be selected $\left(0.007<\varepsilon_{y}<0.009\right)$ than 0.3 value identified in the case of transversal vibrations only [1], whereas for the lower mass ration coupling coefficient $A_{y}$ should be reduced, but for the higher mass ratio, it should be increased in comparison with 12 value from [1].

\subsection{Calibration using CFD modelling}

As was mentioned earlier, experimental facilities are different from each other and having access to these facilities is not always possible. In order to use the experimental data for the model calibration, it would be useful to refine the proposed generic model in order to accommodate the specific features of the chosen experimental rig. In such cases, it is inevitable that the additional effects would complicate the main phenomenon and might make it challenging to separate the core vortex induced vibrations from structural nonlinear vibrations of the rig, for example. Therefore, we will explore the CFD approach to calibrate the proposed 2DOF wake oscillator model. The appropriate CFD model could be set up without extra structural 
(a)

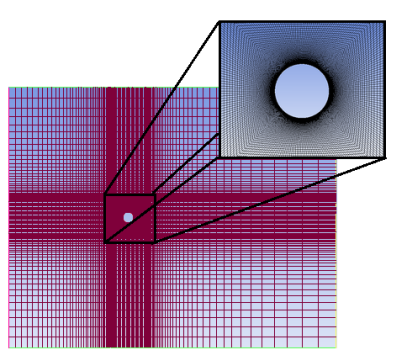

(b)

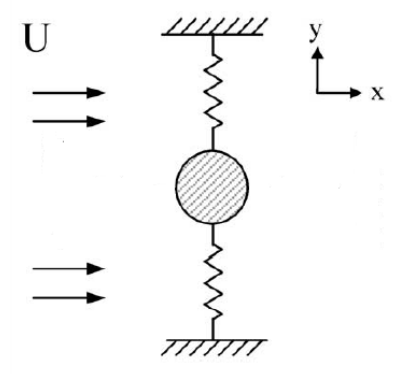

(c)

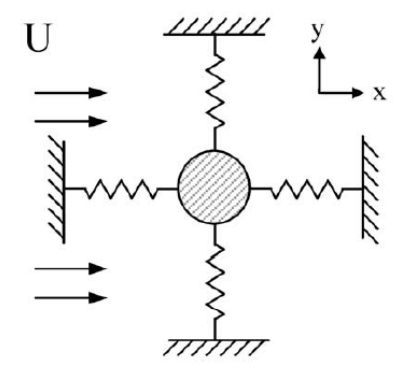

Figure 9: (colour online) (a) Computational mesh showing a zoom-up of the area near a cylinder; (b) a single degree-of-freedom system [17] and (c) a two degrees-of-freedom system [17].

complications and if it is properly verified, such a CFD model could be a very valuable tool for the wake oscillator model calibration.

\subsubsection{CFD model}

In this section, we consider the behaviour of elastically supported cylinder capable of moving in in-line and transversal directions (often referred in literature as XY motion).

CFD model [27] has been implemented in ANSYS Fluent 12.0.16 utilizing User Defined Functions (UDFs) in order to compute the displacement of the cylinder on each time step based on the forces obtained from the dynamic pressure. Relatively low Reynolds numbers (600 to 2000) were considered for the sake of simplicity. However, even for these Reynolds numbers $(R e>300)$ the vortex street is turbulent, and a high quality grid is required for solution to converge.

To couple the motions of the cylinder and the fluid, the forces acting on the cylinder have been calculated by integrating the total wall pressure on the cylinder surface obtained from the CFD solver, and the drag and lift coefficients have been obtained as non-dimensional components of these forces.

The computational domain used in this study is shown in Fig. 9a, where a cylinder of a unit diameter was considered [27]. The domain consists of an upstream of 11.5 times the diameter to downstream of 20 times the diameter of the cylinder and 12.5 times on each cross stream direction. The data grid contains 15380 quadrilateral cells and 15659 nodes (Fig. 9a). A finer grid is created near the boundary layer around the cylinder and it gets coarser at far flow field, particularly outside of the wake region. The inlet region is on the left side and the outlet is on the right side of the grid and periodic boundary conditions were chosen for the upper and lower boundaries of the computational domain. The PISO (Pressure Implicit solution by Split Operator method) pressure-velocity coupling scheme [28] was used as a solution method to allow a larger time step size without compromising the stability of the solution.

Dynamic mesh operated by the UDF allows motion of the cylinder in a two-dimensional plane. The spring-based smoothing method [28] is applied to all cells of the dynamic mesh. The UDF receives full feedback from the fluid and controls the displacement of the cylinder by incorporating the 'Compute Force and Moment' function into the equation of motion, providing lift and drag forces directly from the solver on each time step. The time step size was chosen with regards to the Strouhal number appropriate for shedding frequency (defined as the frequency of a complete vortex shedding cycle), stream velocity and diameter of 
the cylinder. To capture the vortex shedding correctly, at least 50 time steps were performed in one shedding cycle which was calculated as $T_{\text {cycle }}=D / S t U$. At the start of the simulations, the cylinder is at rest in its initial equilibrium position, and the initial conditions for cylinder's transverse and in-line displacements and velocities are $y(0)=0$ and $\dot{y}(0)=0$, and $x(0)=0$ and $\dot{x}(0)=0$.

The first set of simulations was carried out for the cylinder capable of moving in transverse direction only, and amplitudes of the oscillations were recorded under varying the flow velocity. In this case the equation of motion of the cylinder is coupled with the fluid simulations via lift coefficient which is calculated by CFD on each time step of the simulation process. The equation of motion for the transversal displacement $y$ is

$$
m_{s} \ddot{y}+r_{s} \dot{y}+h y=\frac{1}{2} \rho_{f} D U^{2} C_{Y}(t),
$$

where $m_{s}$ is mass per unit length, $C_{Y}(t)$ is the non-dimensional lift coefficient obtained from CFD solver using the transversal (lift) fluid force as $C_{Y}(t)=2 F_{Y}(t) /\left(\rho_{f} D U^{2}\right)$. A dot denotes the differentiation with respect to dimensional time $t$.

The second set of simulation was run for the cylinder moving in both transversal and in-line directions. The equation of motion for the in-line displacement $x$ is

$$
m_{s} \ddot{x}+r_{s} \dot{x}+h x=\frac{1}{2} \rho_{f} D U^{2} C_{X}(t),
$$

where $C_{X}(t)$ is drag coefficient obtained from the CFD solver in a similar manner.

\subsection{2. $C F D$ Results}

In this sub-section, we present the comparison of the results for vibrations of a single degree-of-freedom system (transversal vibration of the rigid cylinder) and a two degrees-of-freedom system (transversal and in-line vibrations of the cylinder). While the natural frequency of the structure is kept constant in order to fix structural properties, a variation of reduced velocity, $U_{r e d}=2 \pi U /\left(\omega_{n} D\right)$, is undertaken by altering the flow velocity, $U$. In this case Reynolds number is not a constant value and the solution quality is heavily dependent on and constrained by the quality of the grid. The calculations were performed for the damping ratio $\zeta=r_{s} /\left(m_{s} 2 \omega_{n}\right)=0.01$ and various mass ratios $m^{*}=4 m_{s} /\left(\rho_{f} \pi D^{2}\right)$. The results are presented for the displacement which is normalised with respect to the diameter of the cylinder, $D$.

It is well known that the frequency of the cylinder's vibrations is directly related to the vortex shedding frequency and that when the flow velocity generates the vortex shedding at the frequency close to the cylinder's natural frequency $\omega_{n}$, the shedding frequency locks onto it, and the lock-in resonant oscillations occur at or near the natural frequency of the structure and tend to have significantly greater amplitude. In the current study the entry to and exit from the lock-in condition were considered in the range of reduced velocities from approximately 3 to 10 (or Reynolds numbers from 600 to 2000).

The system behaviour at various mass ratios was investigated, and particular attention was paid to lowmass ratio cylinders. Fig. 10a presents the amplitude of the transversal vibration as a function of reduced velocity for two different values of cylinder's mass ratio $m^{*}=1.275$ and 6.375 where cylinder is allowed to move in both directions. As can be seen in the figure, lower mass ratio cylinder demonstrates significantly larger peak value of dimensionless amplitude. In this case, the CFD simulations reveal that the drag force 
(a)

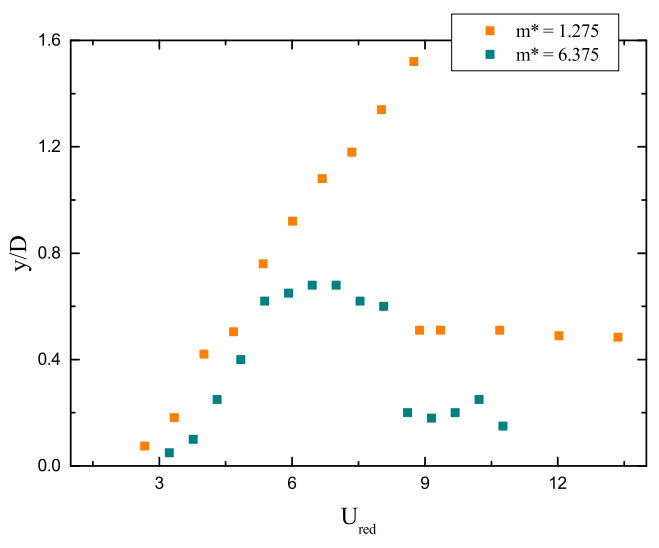

(b)

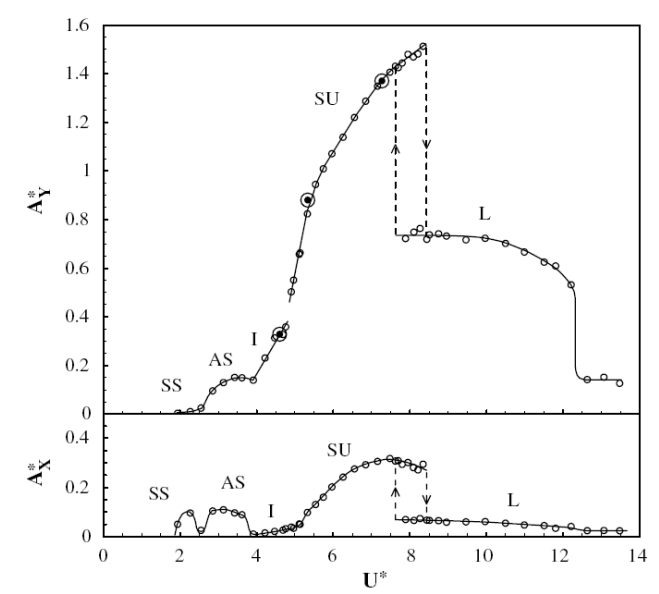

Figure 10: (colour online) (a) Amplitude response at two different mass ratios $m_{*}=1.275$ and $m_{*}=6.375$ calculated using CFD, with a "super-upper" branch appearing at a lower mass ratio $m_{*}=1.275$, [29]; (b) "Super-upper" branch obtained experimentally [26].

(a)

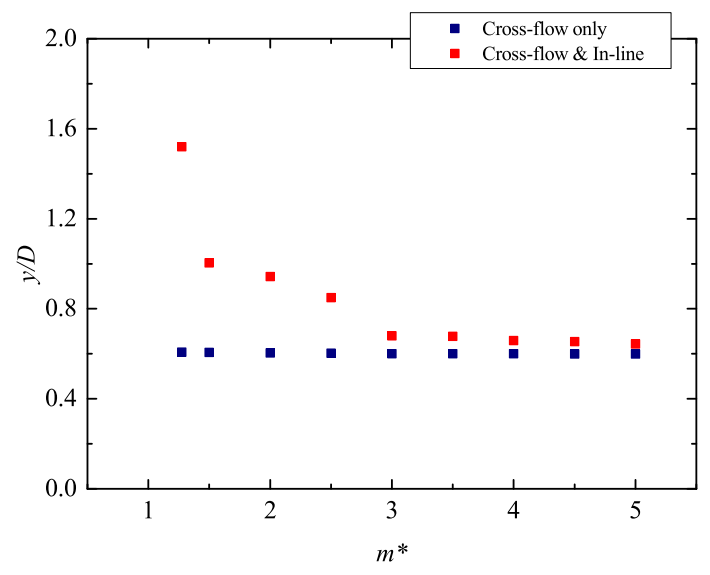

(b)
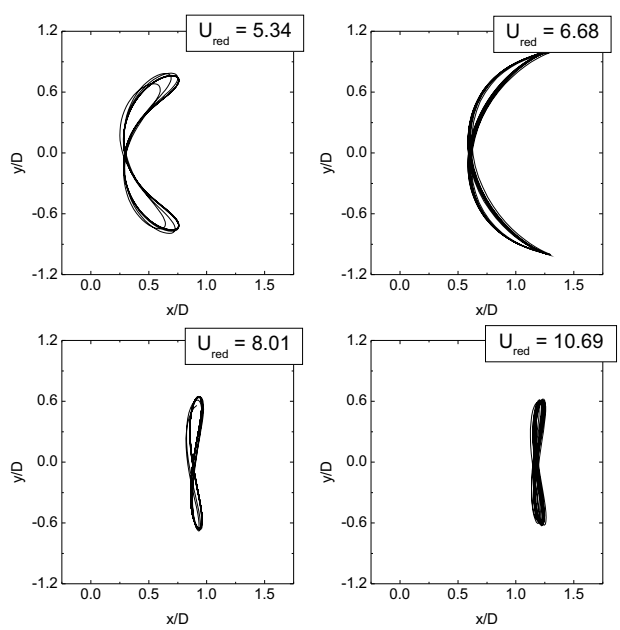

Figure 11: (colour online) (a) Comparison of 1DOF and 2DOF amplitudes of non-dimensional cylinder displacement at various mass ratios [29]; (b) Trajectories of the cylinder motion on the phase plane for $m^{*}=1.275$ for four different values of $U_{\text {red }}=$ $5.34,6.68,8.01$ and 10.69 .

influences cross-flow amplitude, and it is observed that drag's impact at resonance becomes more significant as mass ratio of a cylinder decreases.

Experiments by Williamson and Jauvtis $[17,26]$ demonstrated a drastic increase of amplitude when mass ratio is decreased below $m^{*}=6$ as shown in Fig. 10b. A new branch of response appeared in case of two degrees-of-freedom motion of the structure, and it was defined as a "super-upper" branch. As can be seen from Fig. 10, our computational results agree well with the existence of this branch, although the peak amplitude exhibits lower value than in physical experiments.

Comparison between peak amplitudes of the single degrees-of-freedom cylinder and the two degrees-offreedom cylinder at various mass ratios is made and represented in Fig. 11a. It can be seen that, for the ratios below $m^{*}<3$, the two degrees-of-freedom cylinder demonstrates the presence of "supper-upper" branch. In this case in-line vibrations have significant impact on cross-flow amplitude, and this effects becomes more pronounced as mass ratio decreases. To demonstrate this, the trajectories of the cylinder on $X Y$ plane are shown in Fig. 11b for various values of the reduced velocity at $m^{*}=1.25$. Here two values $U_{\text {red }}=5.34$ and 
(a)

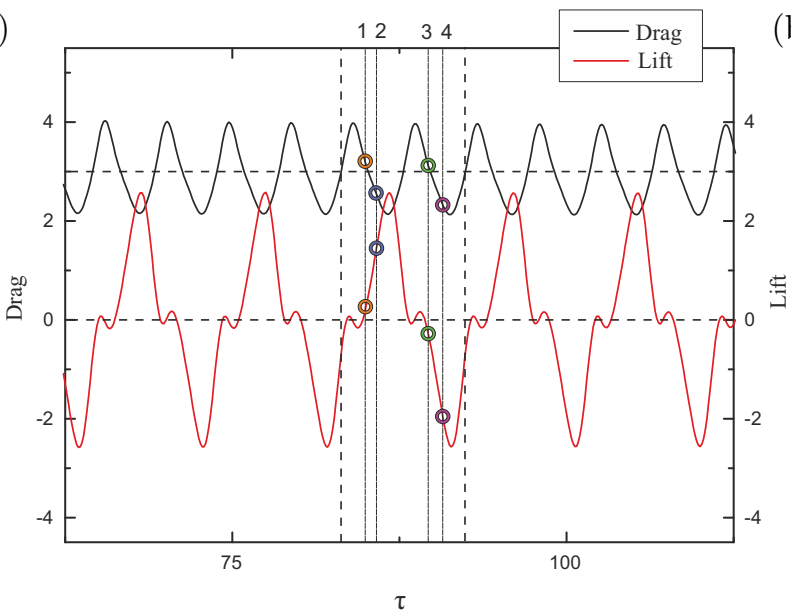

(b)
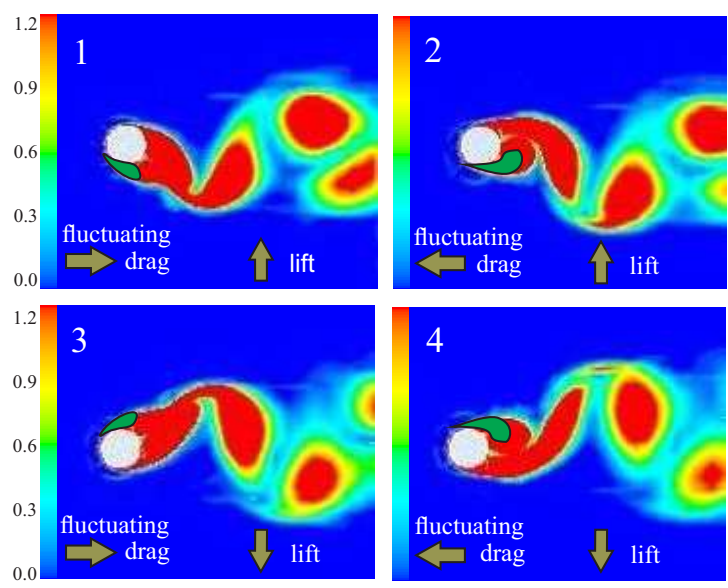

Figure 12: (colour online) (a) Lift and drag history for $m^{*}=1.275$ at $U_{\text {red }}=6.68$ (lock-in condition); the numbers indicate the time moments where the pressure distribution presented in Fig. 12b are calculated; dash lines show the beginning and the end of one cycle; (b) Vorticity contours calculated at different times during one cycle. Grey arrows indicate the direction of vortex-induced lift and drag forces.

6.68 belong to "super-upper" branch and the other two values $U_{\text {red }}=8.01$ and 10.69 are chosen from outside region. As can be seen in Fig. 11b, the trajectory of motion becomes asymmetric to the $\mathrm{Y}$ axis and the original "eight" shape is bent. The increase of amplitude at illustrated reduced velocity values is significant and estimates about $50-60 \%$. At the same time, at higher values of reduced velocities, outside of the lock-in region, the influence of in-line vibrations becomes minimal as can be seen in Fig. 11b where the cross-flow dimensionless amplitude is decreased to 0.6 in the presented case.

In case of $X Y$ motion a frequency doubling phenomenon in in-line response is well-known and observed in experiments [25], and Fig. 12 demonstrates that is also captured properly by the CFD simulations. The time histories of non-dimensional drag and lift forces are shown in Fig. 12a where a steady state response is presented for $m^{*}=1.275$ and $U_{\text {red }}=6.68$. The vorticity contours shown in Fig. 12b are calculated at the times marked by numbers 1 to 4 in Fig. 12a, and here the directions of the fluctuating drag and the lift forces are given by the grey arrows. It is observed that this frequency doubling phenomenon can be explained exclusively through the fluid motion and it is not a property of the structure. As can be seen from Fig. 12b, during one period of motion the in-line component of total pressure is switching its direction every quarter of a vortex-shedding cycle as the flow around the cylinder generates vortex-induced drag force along the stream (before passing the middle of the cylinder) and against the stream (after passing the middle of the cylinder). The cross-flow component of total pressure always acts in one direction per half of a vortex-shedding cycle.

Components of the total hydrodynamic force acting on the cylinder, containing contributions from both the lift and drag, cause the cylinder motion in XY plane. This XY motion is schematically illustrated in Fig. 13. For each position of the cylinder in this figure, a corresponding snapshot of vorticity contours obtained with the CFD simulations of the cylinder vibrating under lock-in condition is shown. It is important to note here that ANSYS Fluent decomposes the total hydrodynamic force into two projections $\left(\vec{F}_{X}\right.$ and $\left.\vec{F}_{Y}\right)$ on $\mathrm{X}$ and $\mathrm{Y}$ axes, which do not normally coincide with the directions of lift and drag forces $\left(\vec{F}_{L}\right.$ and $\vec{F}_{D}$ ) of an oscillating cylinder (although they do for a fixed one). These components $C_{X}(t)$ and $C_{Y}(t)$ of 


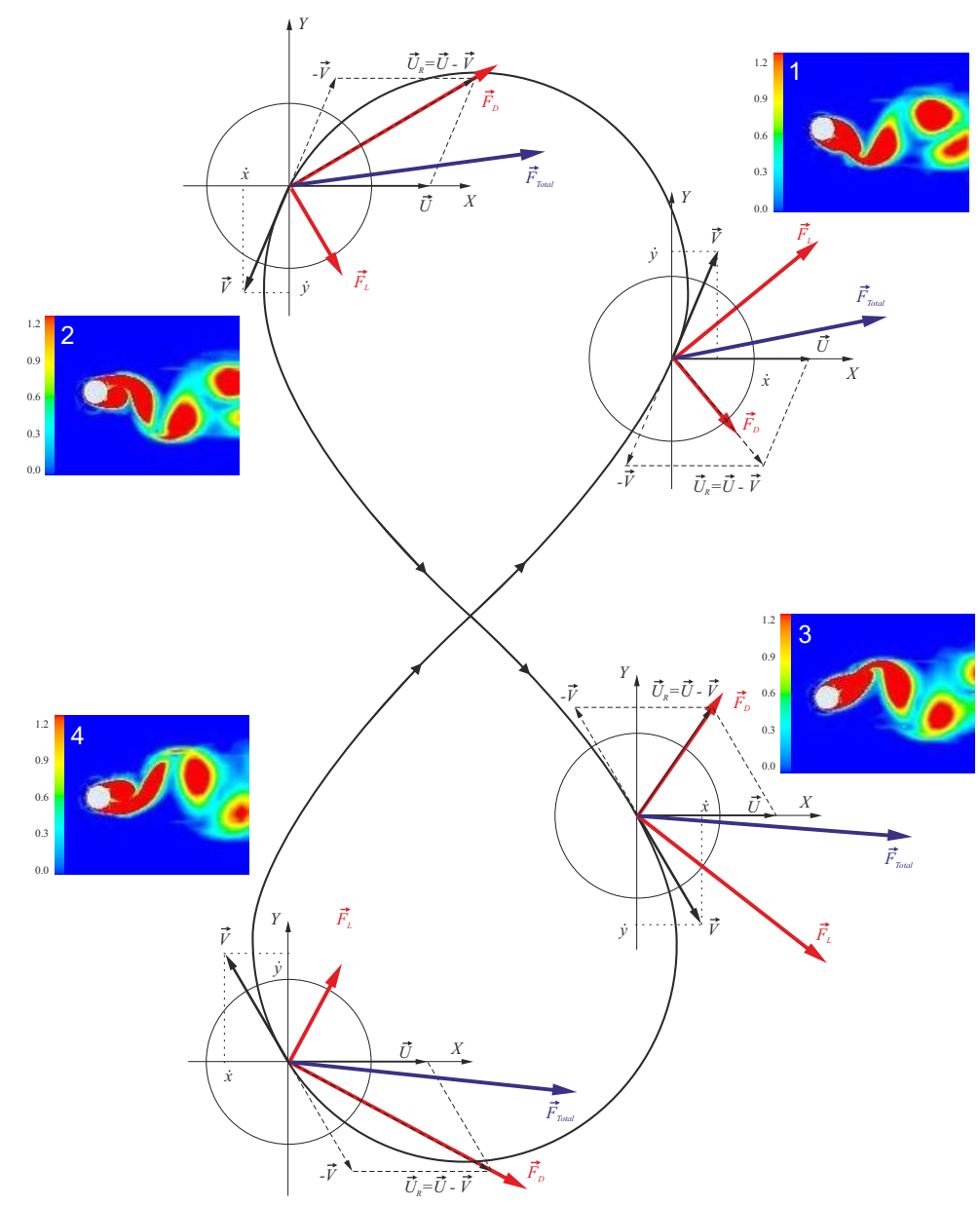

Figure 13: (colour online) Full vortex shedding cycle schematic. The snapshots of the vorticity contours of the cylinder vibrating in the lock-in conditions are presented at four different moments of time during one cycle. In those moments of time the total force acting from the fluid on the cylinder calculated using CFD results for $m^{*}=1.275$ is shown by thick blue arrow. The components of this force $\vec{F}_{\text {Total }}$ acting along the relative fluid flow $\left(\vec{F}_{D}\right)$ and in perpendicular directions $\left(\vec{F}_{L}\right)$ are shown by red thick arrows. Velocities of the cylinder are shown by black solid arrows and schematics to determine the relative flow velocity $\vec{U}_{R}$ are also included.

dimensionless total force computed with the CFD were used to determine the direction of $\vec{F}_{\text {Total }}$, the total hydrodynamic force vector, and to identify for each particular drag force $\vec{F}_{D}$ (see positions 1-4 in Fig. 13) the direction of lift force $\vec{F}_{L}$. While the direction of $\vec{F}_{D}$ coincides with the direction of the relative fluid flow $\vec{U}_{R}$, $\vec{F}_{L}$ acts perpendicularly in one of two possible directions. It can be seen that the total hydrodynamic force vector always falls in I and IV quadrants. In this particular illustration, $C_{Y}(t)$ is in phase with dimensionless cylinder displacement $y / D$.

\subsubsection{Model calibration using CFD results}

The CFD results are used in this sub-section to calibrate the proposed wake oscillator model. Specifically, empirical wake parameters $A_{x}, A_{y}, \varepsilon_{x}, \varepsilon_{y}$ are considered and determined using parametric analysis. Also, fluid force (reference) parameters $C_{L_{0}}, C_{D_{0}}, C_{D_{0}}^{f l}$, taken from a fixed cylinder lift and drag measurements, are discussed here.

It is also important to note, that all presented comparisons are made using mass ratio notation as it appears in the work by Jauvtis and Williamson [17], where $m^{*}=4 m_{s} / \rho_{f} \pi D^{2}$, i.e. the mass ratio does not 

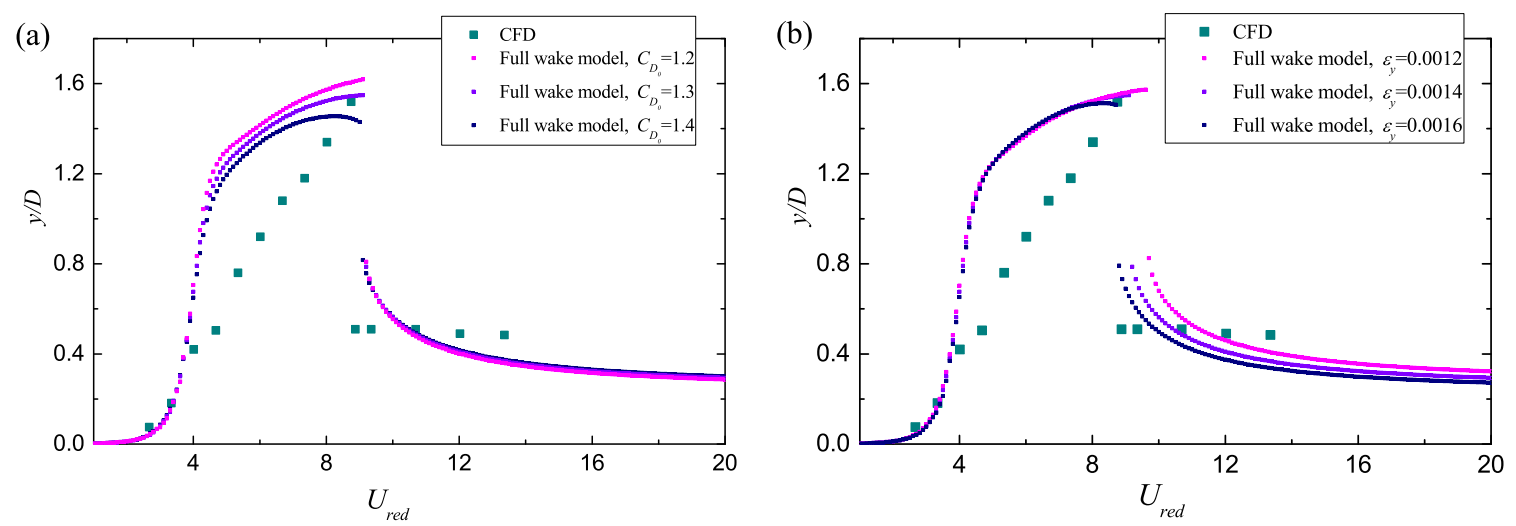

Figure 14: Two degrees-of-freedom: CFD vs Wake Oscillator Model cross-flow response branches for $m^{*}=1.275, \zeta=0.01$. Wake parameters are $A_{y}=3, A_{x}=12, \varepsilon_{x}=0.3$. Reference parameters are $C_{L_{0}}=0.3, C_{D_{0}}^{f l}=0.2$. (a) Effect of mean sectional drag $C_{D_{0}}, \varepsilon_{y}=0.0014$; (b) Influence of wake parameter $\varepsilon_{y}, C_{D_{0}}=1.3$.

include added mass.

For comparisons with data obtained from the CFD simulations, full wake oscillator model, introduced in section 2 and described by Eqs. (37)-(40), is used in its dimensional form

$$
\begin{aligned}
\left(m_{s}\right. & \left.+\frac{1}{4} \pi C_{M} \rho_{f} D^{2}\right) \ddot{x}+r_{s} \dot{x}+h x=\sqrt{(U-\dot{x})^{2}+\dot{y}^{2}}\left(\frac{1}{4} \rho_{f} C_{L_{0}} q D \dot{y}+\right. \\
& \left.+\frac{1}{2} \rho_{f}\left(C_{D_{0}}+\frac{1}{2} C_{D_{0}}^{f l} w\right) D(U-\dot{x})\right) \\
\left(m_{s}\right. & \left.+\frac{1}{4} \pi C_{M} \rho_{f} D^{2}\right) \ddot{y}+r_{s} \dot{y}+h y=\sqrt{(U-\dot{x})^{2}+\dot{y}^{2}}\left(\frac{1}{4} \rho_{f} C_{L_{0}} q D(U-\dot{x})-\right. \\
& \left.-\frac{1}{2} \rho_{f}\left(C_{D_{0}}+\frac{1}{2} C_{D_{0}}^{f l} w\right) D \dot{y}\right)
\end{aligned}
$$

with wake equations

$$
\begin{aligned}
& \ddot{w}+2 \Omega_{F} \varepsilon_{x}\left(w^{2}-1\right) \dot{w}+4 \Omega_{F}^{2} w=\left(A_{x} / D\right) \ddot{x} \\
& \ddot{q}+\varepsilon_{y} \Omega_{F}\left(q^{2}-1\right) \dot{q}+\Omega_{F}^{2} q=\left(A_{y} / D\right) \ddot{y}
\end{aligned}
$$

In general, tuning reference fluid parameters may be hard to justify, and would require a clear explanation since $C_{D_{0}}^{s t}, C_{D_{0}}^{f l}$ and $C_{L_{0}}$ represent experimentally observed force components acting on a fixed cylinder: nonamplified mean sectional drag, fluctuating drag coefficient and fluctuating lift coefficient respectively. All these parameters are usually taken from literature and modifying their values could be questionable. On the other hand, the empirical wake parameters do not have clear physical meaning and they do not necessarily have to be fixed and/or remain the same for different values of mass or damping of the system. Therefore they should be adjusted first when the model calibration is performed.

Comparisons of the results of the simulations obtained using the 2DOF wake oscillator model described by Eqs. (37)-(40) with the 2DOF CFD results are presented in Figs. 14-17. Here the reference parameters for lift and drag coefficients, $C_{L_{0}}$ and $C_{D_{0}}^{f l}$ are kept at their initial values [1], with $C_{L_{0}}=0.3$ and $C_{D_{0}}^{f l}=0.2$.

From Fig. 14a it can be seen that the variation of drag coefficient $C_{D_{0}}$ affects the amplitude of response during lock-in and has almost no effect after desynchronization. At the same time, $\varepsilon_{y}$ primarily affects exit from lock-in when desynchronization takes place as can be seen in Fig. 14b. Increasing $C_{D_{0}}$ leads to lower 
(a)

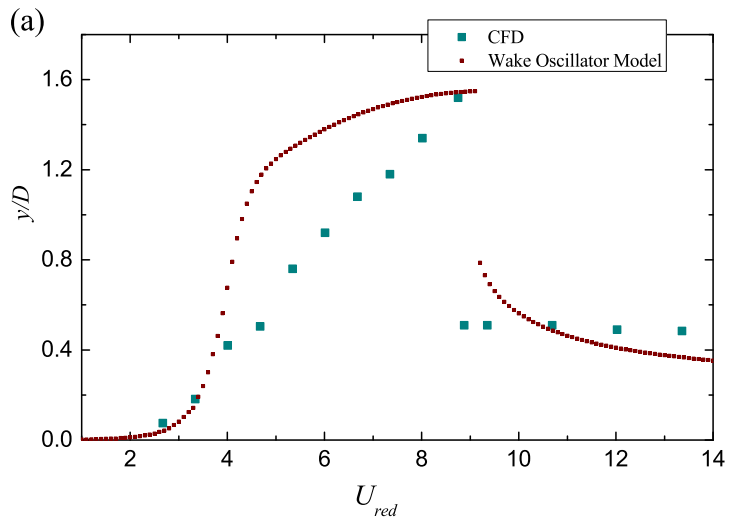

(b)

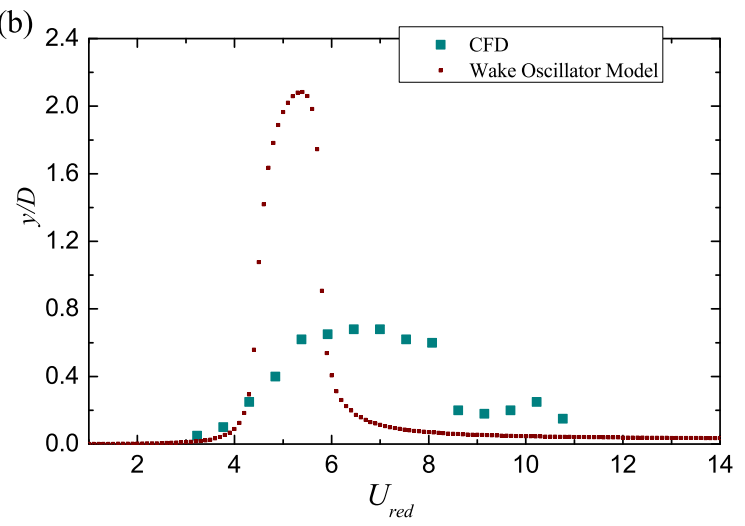

Figure 15: Two degrees-of-freedom: CFD and wake oscillator model cross-flow response branches for (a) $m^{*}=1.275$ and (b) $m^{*}=6.375$. Parameters $C_{D_{0}}=1.3, C_{D_{0}}^{f l}=0.2, C_{L_{0}}=0.3, A_{y}=3, A_{x}=12, \varepsilon_{y}=0.0014, \varepsilon_{x}=0.3$ are fixed for both mass ratios. Damping ratio $\zeta=0.01$.
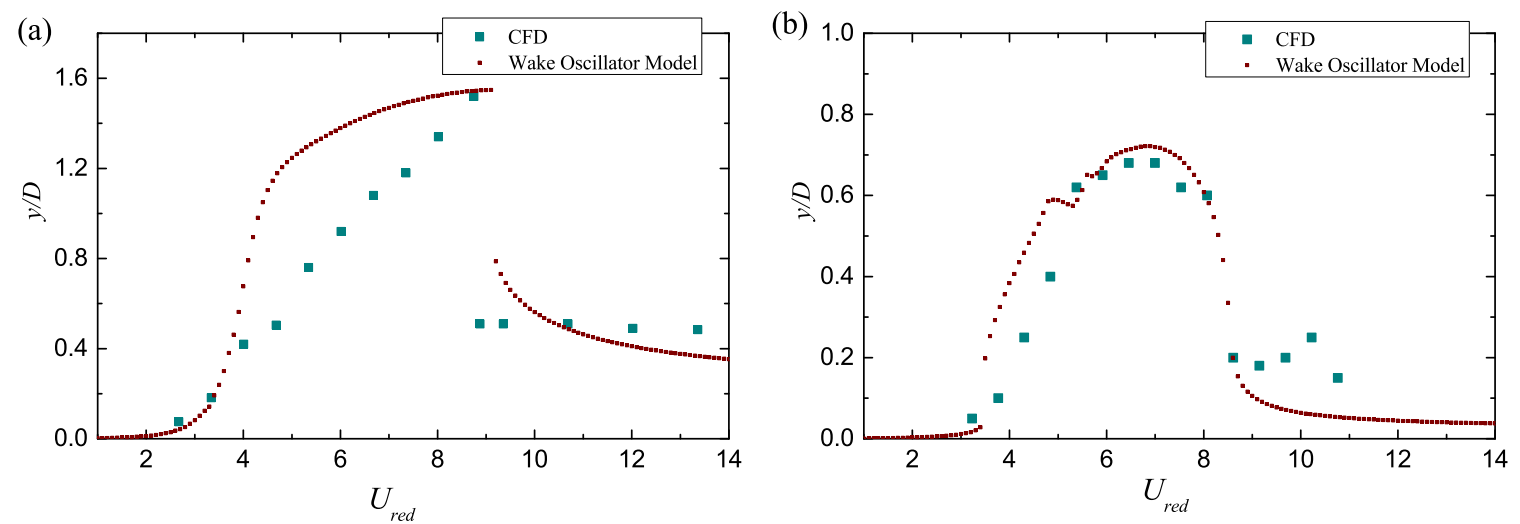

Figure 16: Two degrees-of-freedom: CFD and wake oscillator model cross-flow response branches at initial reference parameters [1] $C_{D_{0}}^{f l}=0.2, C_{L_{0}}=0.3$. (a) $m^{*}=1.275, C_{D_{0}}=1.3, A_{y}=3, A_{x}=12, \varepsilon_{y}=0.0014, \varepsilon_{x}=0.3$; (b) $m^{*}=6.375, C_{D_{0}}=2.0, A_{y}=$ $26, A_{x}=12, \varepsilon_{y}=0.05, \varepsilon_{x}=0.3$. Damping ratio $\zeta=0.01$.

amplitudes in the lock-in region and higher jump, while reducing $\varepsilon_{y}$ leads to wider lock-in region, but has little to no influence on the height of the jump. $A_{x}$ and $\varepsilon_{x}$ were kept at $A_{x}=12$ and $\varepsilon_{x}=0.3$ as appear in [1].

In Fig. 15a the case where parameters $A_{y}, \varepsilon_{y}$ are tuned to demonstrate the best fit with the CFD results for $m^{*}=1.275$ is presented. Although a fairly good correspondence with the CFD results is shown in Fig. 15a for this mass ratio $m^{*}=1.275$, with the same values of reference and wake parameters used for mass ratio $m^{*}=6.375$ in Fig. $15 \mathrm{~b}$ the model fails to predict the amplitudes of the response. Better results can be achieved when these parameters are treated as a function of mass ratio, or, more generally, as a function of mass-damping ratio, instead of being kept constant as shown in Fig. 16. These conclusions have also been drawn by other researches (see [13]).

In Fig. 16 two sets of tuned wake parameters at different values of amplified drag $C_{D_{0}}$ demonstrate a good match with the CFD data for two different mass ratios. It is clear that a more detailed study should primarily focus on the combined $A_{y} / \varepsilon_{y}$ and $A_{x} / \varepsilon_{x}$ parameters as a function of the mass-damping parameter $\left(m^{*}+C_{M}\right) \zeta$. As can be seen in Fig. 16a, tuned wake parameters for $m^{*}=1.275$ are $A_{y}=3$ and $\varepsilon_{y}=0.0014$, 

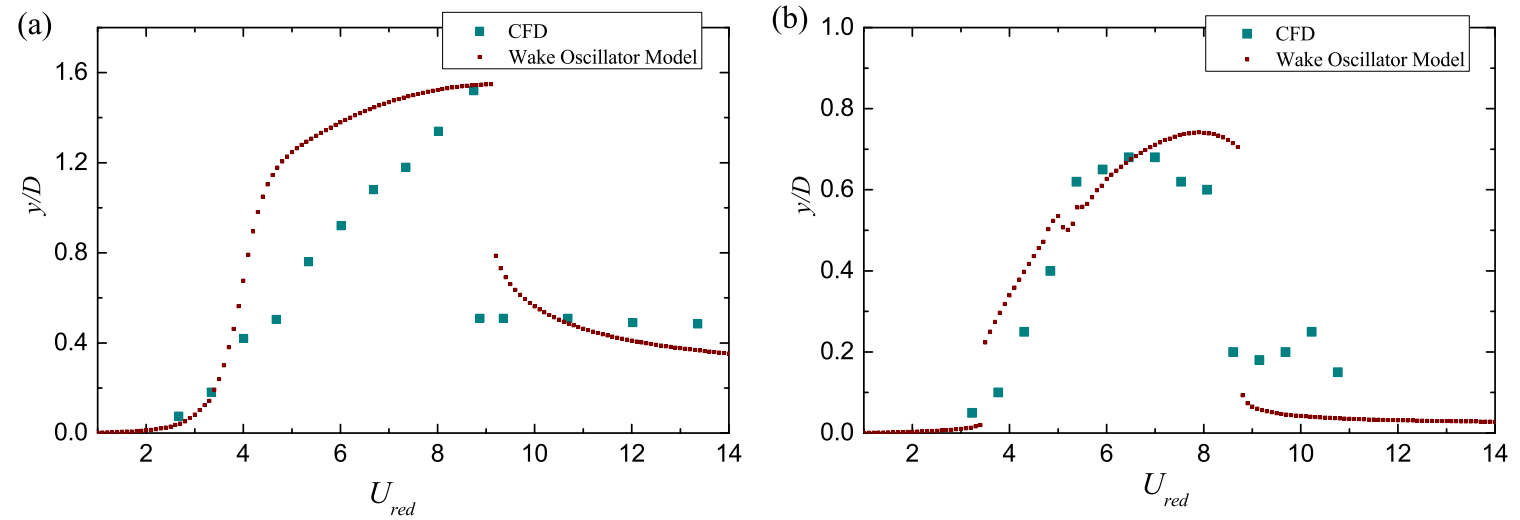

Figure 17: Two degrees-of-freedom: CFD and wake oscillator model cross-flow response branches at initial reference parameters [1] $C_{D_{0}}^{f l}=0.2, C_{L_{0}}=0.3$. (a) $m^{*}=1.275, C_{D_{0}}=1.3, A_{y}=3, A_{x}=12, \varepsilon_{y}=0.0014, \varepsilon_{x}=0.3$; (b) $m^{*}=6.375, C_{D_{0}}=1.3, A_{y}=$ $24, A_{x}=12, \varepsilon_{y}=0.2, \varepsilon_{x}=0.3$. Damping ratio $\zeta=0.01$.
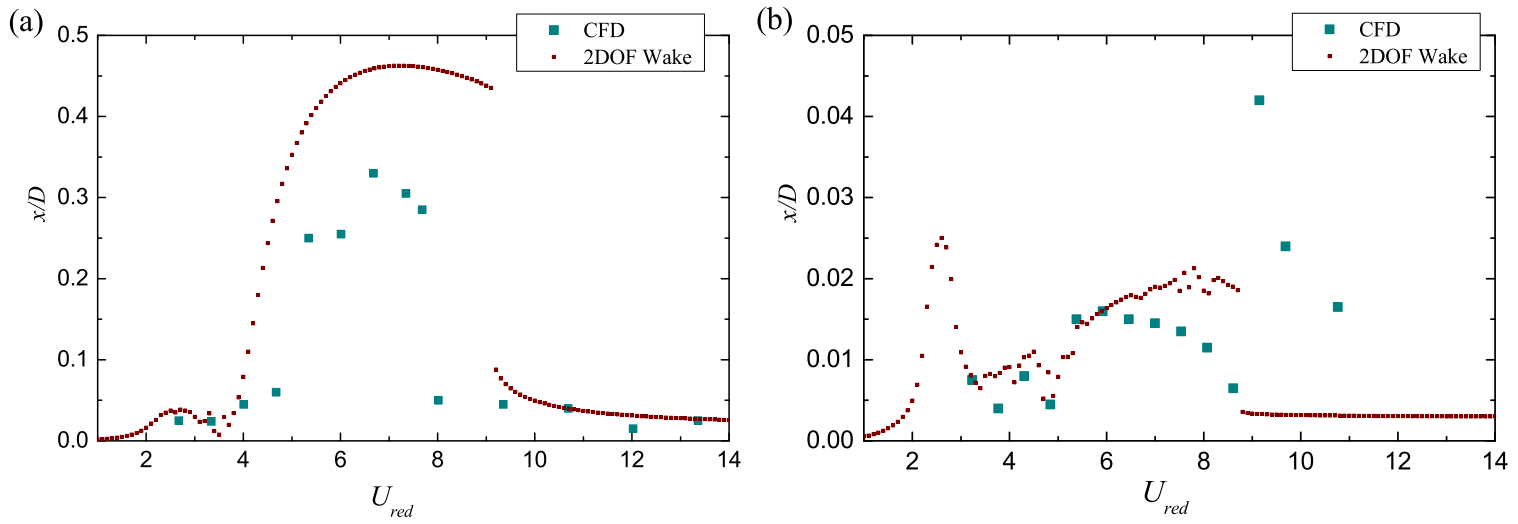

Figure 18: Two degrees-of-freedom: CFD and wake oscillator model in-line response branches at initial reference parameters [1] $C_{D_{0}}^{f l}=0.2, C_{L_{0}}=0.3$. (a) $m^{*}=1.275, C_{D_{0}}=1.3, A_{y}=3, A_{x}=12, \varepsilon_{y}=0.0014, \varepsilon_{x}=0.3 ;$ (b) $m^{*}=6.375, C_{D_{0}}=1.3, A_{y}=$ $24, A_{x}=12, \varepsilon_{y}=0.2, \varepsilon_{x}=0.3$. Damping ratio $\zeta=0.01$.

which give us $A_{y} / \varepsilon_{y}=2143$. For a higher value of mass ratio $m^{*}=6.375$, as illustrated in Fig. 16b, a good match with CFD results is achieved at $A_{y}=26$ and $\varepsilon_{y}=0.05$, with $A_{y} / \varepsilon_{y}=520$. However, at the higher mass ratio $m^{*}=6.375$ the wake oscillator model does not capture a jump from the upper to the lower branch of response.

Different tuning is shown in Fig. 17 with the same value of amplified drag $C_{D_{0}}=1.3$ for both masses. As can be seen in Fig. 17a, wake parameters are the same as they were in the previous figure for $m^{*}=1.275$ with $A_{y}=3$ and $\varepsilon_{y}=0.0014$. For a higher value of mass ratio $m^{*}=6.375$ shown in Fig. $17 \mathrm{~b}$, wake parameters are changed to $A_{y}=24$ and $\varepsilon_{y}=0.2$, with $A_{y} / \varepsilon_{y}=120$. Although amplified drag would generally be different for different masses as it depends on the value of amplitude, for simplicity, when it is taken as constant, it is taken so for all values of mass ratio. In can also be noted that for this set of parameters the jump from the upper to the lower branch is now captured for $m^{*}=6.375$ (Fig. 17b), although amplitude values predicted by wake oscillator model after the jump are less than those predicted by CFD.

Finally, in-line response predictions are shown in Fig. 18. All parameters are the same as for the previous cross-flow responses, and are also presented for $m^{*}=1.275$ (Fig. 18a) and $m^{*}=6.375$ (Fig. 18b). As was 
mentioned before, it has been found that wake parameters $A_{x}$ and $\varepsilon_{x}$ have much smaller effect on in-line amplitude predictions than $A_{y}$ and $\varepsilon_{y}$ have on cross-flow amplitudes. As can be seen from both figures, predictions by wake oscillator model do not capture the in-line response amplitudes as good as they do the cross-flow ones. The values of the in-line amplitudes, however, are significantly lower than those for cross-flow motion, and often negligible, especially for higher mass ratios. It should be noted that overestimation of the in-line amplitudes would be acceptable from the design point of view as the safe solution will be developed based on the prediction of this model.

\section{CONCLUDING REMARKS}

In this work, vortex-induced vibrations of elastically supported cylinders capable of moving in cross-flow and in-line directions were investigated. A new wake oscillator model for two degrees-of-freedom vortexinduced vibrations has been proposed, where vortex-induced lift and drag forces were modelled with two nonlinear self-excited oscillators of van der Pol type.

Total hydrodynamic force was represented as a sum of lift and drag forces acting on a vibrating cylinder in a uniform flow. The lift and drag forces were defined as being proportional to the square of the magnitude of the relative flow velocity around the cylinder with the drag force acting in the direction of relative velocity and lift force acting perpendicularly. Equations of motion of the cylinder in cross-flow and in-line directions are coupled through the fluid forces calculated from the instantaneous relative flow velocity around the cylinder that depends both on the fluid flow velocity and the instantaneous velocity of the cylinder; the type of coupling for a two degrees-of-freedom wake oscillator model that has not been done before. An acceleration coupling was used based on the recommendations by Facchinetti et al. [1].

It was demonstrated that approximation of the fluid forces allows to obtain the well-known low-dimensional models in the limit case, and the model proposed by Facchinetti et al. [1] was used an example of the model reduction to a single degree-of-freedom system. A two-dimensional CFD model developed as a part of this work was used together with the published experimental data to calibrate the wake oscillator model parameters.

It was obtained that to achieve a reasonable match with the experiments much lower values of the parameter $\varepsilon_{y}$ should be selected (for the proposed model, as presented in this work, $0.007<\varepsilon_{y}<0.009$ ) than 0.3 value identified in the case of transversal vibrations only [1], whereas for the lower mass ratios coupling coefficient $A_{y}$ should be reduced, and for the higher mass ratios, it should be increased in comparison with 12 value from [1]. It was also found that the variation of drag coefficient $C_{D_{0}}$ affects the amplitude of response during lock-in and have almost no effect on the predicted length of the lock-in region, whereas $\varepsilon_{y}$ primarily affects this length. Increasing $C_{D_{0}}$ leads to lower amplitudes in the lock-in region and higher jump, while reducing $\varepsilon_{y}$ leads to wider lock-in region, but has little to no influence on the hight of the jump.

Our analysis has shown that the wake oscillators parameters depend on the mass ratio as the best agreement with the experiments is observed for different values of these parameters at low and high mass ratios. In the considered cases for the chosen system parameters, the model slightly overestimated the in-line amplitudes of the vibrations and also amplitudes of the transversal vibrations in the beginning of the lock-in region. It was observed that adjusting parameters of the wake oscillator equation in in-line direction $A_{x}$ 
and $\varepsilon_{x}$ does not have the same effect on the in-line vibration amplitudes as parameters $A_{y}$ and $\varepsilon_{y}$ on the transversal amplitudes.

It should be noted here that although general recommendations on the choice of empirical wake parameters can be given based on this study, in the future a development of some type of optimisation procedure for in-depth model calibration would be beneficial.

More accurate estimation of the amplified drag coefficient $C_{D_{0}}$ has to be done and is also planned for the future work. Instead of taking it as constant, amplified drag can be represented in the form $\left(1+2 A_{y} / D\right) C_{D_{0}}^{s t}$ [30], where $A_{y}$ is the cross-flow amplitude of vibration and $C_{D_{0}}^{s t}$ is the drag coefficient for a stationary cylinder in the subcritical range of Reynolds numbers. This will introduce an additional nonlinearity into structural equations and contribute to refinement of the proposed model.

\section{ACKNOWLEDGMENTS}

This work is supported by the National Subsea Research Institute (NSRI) UK.

[1] Facchinetti M.L., de Langre E., Biolley F., 2004, "Coupling of Structure and Wake Oscillators in VortexInduced Vibrations", Journal of Fluids and Structures 19, 123-140.

[2] Bishop, R.E.D., Hassan, A.Y., 1964, "The Lift and Drag Fores on a Circular Cylinder in a Flowing Field", Proc. Roy. Soc. (London) Ser. A, 277, 51-75.

[3] Skop, R. A., Griffin, O. M., 1973, "A Model for the Vortex-Excited Resonant Vibrations of Bluff Bodies", J. Sound and Vibration 27, 225-233.

[4] Iwan, W.D., Blevins R.D., 1974, "A Model for Vortex-Induced Oscillation of Structures", J. Appl. Mech. $41,581-586$.

[5] Balasubramanian, S., Skop, R.A., 1997, "A New Twist on an Old Model for Vortex-Excited Vibrations", Journal of Fluids and Structures 11, 395-412.

[6] Krenk, S., Nielsen, S.R.K., 1999, "Energy Balanced Double Oscillator Model for Vortex-Induced Vibrations", ASCE Journal of Engineering Mechanics 125, 263-271.

[7] Mureithi, N.W., Kanki, H., Nakamura, T., 2000, "Bifurcation and Perturbation Analysis of Some Vortex Shedding Models", Proceedings of the Seventh International Conference on Flow-Induced Vibrations, Luzern, Switzerland. Balkema, Rotterdam, pp. 61-68.

[8] Plaschko, P., 2000, "Global Chaos in Flow-Induced Oscillations of Cylinders", Journal of Fluids and Structures 14, 883-893.

[9] Skop, R.A., Luo, G., 2001, "An Inverse-Direct Method for Predicting the Vortex-Induced Vibrations of Cylinders in Uniform and Nonuniform Flows", Journal of Fluids and Structures 15, 867-884.

[10] Keber, M., Wiercigroch, M., 2008, "Dynamics of a Vertical Riser with Weak Structural Nonlinearity Excited by Wakes", Journal of Sound and Vibration 315, 685-699. 
[11] Ogink, R.H.M., Metrikine, A.V., 2010, "A Wake Oscillator with Frequency Dependent Coupling for the Modeling of Vortex-Induced Vibration", Journal of Sound and Vibration 329, 5452-5473.

[12] Ge, F., Long, X., Wang, L., Hong Y.Sh., 2009, "Flow-Induced Vibrations of Long Circular Cylinders Modeled by Coupled Nonlinear Oscillators", Science in China Series G: Physics, Mechanics and Astronomy, vol. 52, no. 7, 1086-1093.

[13] Srinil, N., Zanganeh, H., 2012, "Modelling of Coupled Cross-Flow/In-Line Vortex-Induced Vibrations using Double Duffing and Van Der Pol Oscillators", Ocean Engineering 53, 83-97.

[14] Furnes G.K., Sorensen K., 2007, "Flow Induced Vibrations Modeled by Coupled Non-Linear Oscillators", Proceedings of the 17th International Offshore and Polar Engineering Conference, Lisbon, Portugal. 2781-2787.

[15] Wang X.Q., So, R.M.C., Chan, K.T. 2003, ”A Nonlinear Fluid Force Model for Vortex-Induced Vibration of an Elastic Cylinder", Journal of Sound and Vibration 260, 287-305.

[16] Raj, S.P., Rajasekar, S., 1997, "Migration control in two coupled Duffing oscillators", Phys. Rev. E 55 (5), 6237-6240.

[17] Williamson, C.H.K., Jauvtis, N., 2004, "A Hight-Amplitude 2T Mode of Vortex-Induced Vibration for a Light Body in XY Motion", European Journal of Mechanics B/Fluids 23, 107-114.

[18] Stappenbelt, B., Lalji, F., Tan, G. 2007, "Low mass ratio vortex-induced motion", The 16th Australian Fluid Mechanics Conference, Gold Coast, Australia, pp. 1491-1497.

[19] Blevins, R.D., Coughran, C.S. 2009, "Experimental investigation of vortex-induced vibration in one and two dimensions with variable mass, damping, and Reynolds number", Journal of Fluids Engineering 131 (10), 101202-101207.

[20] Xu Bai, Wei Qin, 2014, "Using vortex strength wake oscillator in modelling of vortex induced vibrations in two degrees of freedom". European Journal of Mechanics B/Fluids 48, 165-173.

[21] Vandiver, J.K., 1993, "Dimensionless Parameters Important to the Prediction of Vortex-Induced Vibration of Long, Flexible Cylinders in Ocean Currents", Journal of Fluids and Structures 7, 423-455.

[22] Williamson, C.H.K., Jauvtis, N., 2004, "The effect of two degrees of freedom on vortex-induced vibration at low mass and damping", J. Fluid Mech. 509, 23-62.

[23] Pavlovskaia, E., Keber, M., Postnikov, A., Reddington, K. and Wiercigroch, M. 2016, "Multi-mode modelling of vortex-induced vibration", International Journal of Nonlinear Mechanics 80, 40-51.

[24] Hartlen, R.T., Currie, I.G., 1970, "Lift-Oscillator Model of Vortex-Induced Vibration", Journal of the Engineering Mechanics Division EM5, 577-591.

[25] Vandiver, J.K., Jong, J.-Y., 1987, "The Relationship Between In-Line and Cross-Flow Vortex-Induced Vibration of Cylinders", Journal of Fluids and Structures, 381-399. 
[26] Williamson, C.H.K., Govardhan, R., 2004, "Vortex-Induced Vibrations", Annual Reviews, Fluid Mechanics.

[27] Postnikov, A., 2016, "Wake Oscillator and CFD in Modelling of VIVs", PhD Thesis, School of Engineering, University of Aberdeen.

[28] ANSYS FLUENT 12.0"Theory Guide", ANSYS Inc. April 2009.

[29] Postnikov, A., Pavlovskaia, E.E., Wiercigroch, M., 2012, "Modelling of vortex-induced vibrations of slender marine structures", Proceedings of the 10th International Conference on Flow-Induced Vibration \& Flow-Induced Noise (FIV2012). Flow-Induced Vibration eds. Meskell, C. \& Bennett, G., pp. 569-576.

[30] Pantazopoulos, M.S., 1994, "Vortex-induced vibration parameters: A critical review", OMAE - Volume I, Offshore Technology, ASME, 199-255. 